\section{State of the Art Review}

\section{Check for updates}

\section{OPEN ACCESS}

Received: Oct 14, 2020

Accepted: Oct 27, 2020

Correspondence to

Saurabh Kumar, BSc/MBBS, PhD

Department of Cardiology, Westmead

Hospital, Westmead Applied Research Centre,

University of Sydney, Darcy Road, Westmead,

New South Wales 2145, Australia.

E-mail: saurabh.kumar@sydney.edu.au

Copyright (c) 2021. The Korean Society of Cardiology

This is an Open Access article distributed under the terms of the Creative Commons Attribution Non-Commercial License (https:// creativecommons.org/licenses/by-nc/4.0) which permits unrestricted noncommercial use, distribution, and reproduction in any medium, provided the original work is properly cited.

ORCID iDs

Timothy Campbell (D)

https://orcid.org/0000-0003-4793-7741

Richard G. Bennett (D)

https://orcid.org/0000-0001-9823-3993

Yasuhito Kotake, (D)

https://orcid.org/0000-0003-4690-8885

Saurabh Kumar (D)

https://orcid.org/0000-0002-5643-5076

\section{Conflict of Interest}

This work was supported in part from the NSW Early-Mid Career Fellowship. Kumar S has received research grants from Abbott Medical and Biotronik; he has received honoraria from Biosense Webster, Inc., Abbott Medical, Biotronik, and Sanofi Aventis. Campbell T has received speakers Honoria from Biosense Webster, Inc.

\section{Updates in Ventricular Tachycardia Ablation}

\author{
Timothy Campbell (D, BSc ${ }^{1,2}$, Richard G. Bennett (D), BSc, MBChB ${ }^{1,2}$, \\ Yasuhito Kotake (D, MD, PhD ${ }^{1,2}$, and Saurabh Kumar (D, BSc/MBBS, PhD ${ }^{1,2}$ \\ 'Department of Cardiology, Westmead Hospital, Sydney, Australia \\ ${ }^{2}$ Westmead Applied Research Centre, University of Sydney, New South Wales, Australia
}

\begin{abstract}
Sudden cardiac death (SCD) due to recurrent ventricular tachycardia is an important clinical sequela in patients with structural heart disease. As a result, ventricular tachycardia (VT) has emerged as a major clinical and public health problem. The mechanism of VT is predominantly mediated by re-entry in the presence of arrhythmogenic substrate (scar), though focal mechanisms are also important. Catheter ablation for VT, when compared to standard medical therapy, has been shown to improve VT-free survival and burden of device therapies. Approaches to VT ablation are dependent on the underlying disease process, broadly classified into idiopathic (no structural heart disease) or structural heart disease (ischemic or non-ischemic heart disease). This update aims to review recent advances made for the treatment of VT ablation, with respect to current clinical trials, peri-procedure risk assessments, pre-procedural cardiac imaging, electro-anatomic mapping and advances in catheter and non-catheter based ablation techniques.
\end{abstract}

Keywords: Ventricular tachycardia; Ventricular arrhythmias; Radiofrequency ablation; Multimodal imaging; Sympathectomy

\section{INTRODUCTION}

Sudden cardiac death (SCD) due to recurrent ventricular tachycardia (VT) is an important clinical sequela in patients with structural heart disease (SHD). VT has emerged as a major clinical and public health problem. VT generally occurs as a result of re-entry in the presence of arrhythmogenic substrate (scar), though focal mechanisms remote to scar are prevalent. ${ }^{1)}$ Scar tissue forms due to an ischemic cardiomyopathy (ICM) from prior coronary obstructive disease or a non-ischemic cardiomyopathy (NICM) from an inflammatory, valvular or genetic disease, amongst other reasons. ${ }^{2}$ Critical components of VT circuits related to ICM VT are usually present subendocardially with variable transmural extension. In contrast critical components of VT in NICM can be found in the intramural septum, epicardial surface, and/ or subendocardially.

Treatment for VT includes implantable cardioverter defibrillator (ICD) implantation, antiarrhythmic drug (AAD) therapy and catheter ablation (CA). ICDs prevent cardiac arrest and 
Author Contributions

Conceptualization: Campbell T; Writing - original draft: Campbell T, Bennett RG, Kotake Y, Kumar S; Writing - review \& editing: Campbell T, Bennett RG, Kotake Y, Kumar S. sudden death from VT, but do not stop VT from occurring in the first place. Recurrent VT and ICD therapies decrease quality of life (QOL), increase hospital visits, mortality, morbidity and risk of death. ${ }^{4-6)} \mathrm{AADs}$ can reduce VT recurrence, ${ }^{7)}$ but have significant limitations in treatment of VT in NICM. For example, amiodarone has high rates of side effects/toxicities and limited long term efficacy before drug intolerance due to end organ effects or drug failure ensues. ${ }^{8)}$ In NICM substrates, which has a younger demographic population, ${ }^{9110)}$ AADs are unlikely to provide durable control of VT in the intermediate or long term. Treatment of VT with CA as a result is increasing in some countries where the increment is $16.5-18 \%$, per annum. ${ }^{11)} \mathrm{CA}$ is an important treatment option for reduction in ICD shocks and improved mortality.

The purpose of this update is to explore advances in VT ablation, with respect to current clinical trials, tools for peri-procedural risk assessment, advances in pre-procedural cardiac imaging, electro-anatomic mapping (EAM) and in catheter and non-catheter based ablation technology.

\section{ABLATION VERSUS MEDICAL THERAPY}

The recent randomised clinical trial (RCT), Ventricular Tachycardia Ablation or Escalated Drug Therapy (VANISH), ${ }^{12}$ compared CA to escalated AADs in 259 patients with prior myocardial infarction and ICD implantation. CA over a 27.9 \pm 17.1 months follow up period was superior for the primary outcome of death, VT storm or an appropriate ICD shock. In a follow up sub study of VANISH patients, ${ }^{13)}$ patients with amiodarone-refractory VT had an increased rate of ventricular arrhythmias (VA) and mortality than those with sotalolrefractory VT. Notably patients with sotalol-refractory VT receive a similar outcome if they underwent $\mathrm{CA}$ or escalation of AAD therapy to amiodarone. In contrast, patients on amiodarone who were randomised to CA had improved outcomes following CA, versus those who were randomised to escalation beyond amiodarone (addition of mexiletine).

Anderson et al., ${ }^{14)}$ in a meta-analysis, compared the clinical outcomes of CA versus medical therapy in RCTs of patients with SHD, in addition to comparing those to the results from contemporary observational studies. In 8 RCTs, 797 patients were identified and compared to 3,065 patients in 4 observational studies from experienced single centres or multi-centre prospective studies or registries. In the RCT studies, 165 of 419 patients (39\%) developed VT recurrence in the CA group compared to 185 of 378 patients (48\%) in the medical therapy group. There was a statistically significant $22 \%$ risk reduction in the number of patients in the CA group experiencing recurrent VT in the follow-up period (relative risk [RR], 0.78; $95 \%$ confidence interval [CI], 0.64-0.95; $\mathrm{p}=0.01$ ). Electrical storm (ES; including VT and $\mathrm{VF}$ ) were reported in 4 studies and occurred in $17 \%$ of the CA group and $25 \%$ of the medical therapy group. There was a statistically significant $30 \%$ risk reduction in VT storms occurring during follow up ( $R R, 0.70 ; 95 \% \mathrm{CI}, 0.51-0.94 ; \mathrm{p}=0.02$ ). Mortality occurred in $18 \%$ of CA group compared to $19 \%$ in the medical therapy group. There was no significant difference in all-cause mortality between groups ( $R R, 0.92 ; 95 \% \mathrm{CI}, 0.67-1.27$; $\mathrm{p}=0.62$ ). Contemporary observational studies, in comparison to RCTs had a higher proportion of NICM $(49.4 \%)$ and patients who presented with ES prior to CA (33.2\%). Over a mean follow up of 18.2 months VT recurrence $(28.6 \%)$ was lower than the RCTs $(39 \%, \mathrm{p} \leq 0.001)$. The authors identified that prior RCTs are almost exclusively performed in post infarction substrates, and that future RCTs need to address the important question of whether CA is superior to medical therapy in patients with NICM. 


\section{ABLATION OUTCOMES IN NON-ISCHEMIC CARDIOMYOPATHY}

CA in NICM is challenging due to the complexity of the underlying substrate, which tends to be intramural or sub-epicardial in locations. ${ }^{3115)}$ Ablation only from an endocardial approach has worse VT free survival than a combined endo-epicardial approach. Currently there are no exclusive trials in NICM, and as a result, current data is limited to observational retrospective analysis or prospective non-randomised studies.

In a large single centre series, Kumar et al. ${ }^{16)}$ report on long-term outcomes of VT ablation in patients with and without SHD. This consisted of 98 patients with no SHD, 358 patients with ICM and 239 patients with NICM. NICM consisted of idiopathic dilated (55\%), arrhythmogenic right ventricular cardiomyopathy (ARVC; 16\%), sarcoidosis (5\%), valvular $(13 \%)$, congenital $(8 \%)$ and other cardiomyopathies (3.4\%). NICM patients compared to ICM patients were younger and had less left ventricle (LV) dysfunction. Patients with NICM had more ablation procedures and more epicardial ablation than patients with idiopathic or ICM, suggestive of intra-mural substrate which can be difficult to abolish with contemporary radiofrequency ablation (RFA) techniques. Acute success was poorest in NICM (56\%), compared to ICM $(60 \%)$ and idiopathic $(79 \%)$ patients, though only significant when no SHD was compared to SHD patients $(\mathrm{p} \leq 0.001)$. No significant differences in complications between groups was observed. During median follow up of 6 years (interquartile range [IQR], 3-9 years) from last ablation procedure, VA free survival was greater in patients with ICM than $\operatorname{NICM}(54 \pm 4 \%$ vs. $38 \pm 4 \%, p=0.03)$, though ICM patients were poorer in overall survival than NICM patients $(48 \pm 3 \%$ vs. $74 \pm 3 \%, \mathrm{p} \leq 0.001)$.

\section{Idiopathic dilated cardiomyopathy}

Idiopathic dilated cardiomyopathies (IDCM) are caused by genetic (titin, lamin A/C, phospholamban, and desmin) and acquired causes (alcohol consumption, myocarditis, peripartum, drug mediated and auto immune diseases). ${ }^{17)}$ As a result of the heterogeneity of IDCM, outcomes following ablation have been combined.

In a large single centre series, Muser et al. retrospectively analysed 282 patients with IDCM and recurrent VT. ${ }^{15)}$ Patients were receiving amiodarone (59\%) or sotalol or at least one class I AAD (13\%) before ablation was attempted. A total of 442 procedures were performed (median, 1; range, 1-8) and 36\% of patients required multiple procedures. Multiple VTs (median, 2; IQR, 1-4) were induced during the ablation procedure. Target sites for ablation were the interventricular septum (IVS), aortic cusps and the epicardium. Acute success was achieved in $82 \%$ of patients at final procedure. At a median 48 months (IQR, 19-67 months) of follow up after last procedure, freedom from VT was $79 \%$, heart transplantation occurred in $9 \%$, and death in $15 \%$. Multivariate cox proportionate analysis showed an LV ejection fraction (LVEF) <35\% (hazard ratio HR, 2.6; 95\% CI, 1.06-6.38; $\mathrm{p}=0.037$ ) and inducible VT (HR, 3.53; 95\% CI, 1.42-8.80; $\mathrm{p}=0.007$ ) at post procedure non-invasive programmed stimulation were the only predictors of VT recurrence. Predictors of death/ heart transplantation were New York Heart Association (NYHA) class III/IV (HR, 2.97; 95\% CI, 1.1-7.98; $\mathrm{p}=0.031$ ), LVEF less than 35\% (HR, 4.7; 95\% CI, 1.33-16.55; $\mathrm{p}=0.016$ ) and VT recurrence (HR, 12.12; 95\% CI, 4.58-30.05; $\mathrm{p}<0.001$ ). Muser et al. ${ }^{15)}$ concluded that the majority of patients had good long-term arrhythmia free survival, though up to one third of patients died or required a heart transplantation over follow up. 
Ablation approaches in post infarct VT can either be targeted (entrainment and activation mapping guided) or a complete scar homogenisation (electrical unexcitablity). ${ }^{18199}$ Recently Gokoglan et al. ${ }^{20)}$ in a prospective study reported on a single centre experience in 93 patients with IDCM, monomorphic scar related VT and evidence of low bipolar voltage on EAM. Standard ablation incorporating substrate mapping, activation and entrainment, and pace mapping were compared with an empirically performed endo- and epicardial scar homogenisation. Patients undergoing scar homogenisation had longer RF (21.2 \pm 13.4 vs. $43.64 \pm 37.6$ minutes, $\mathrm{p}<0.001)$ and fluoroscopy $(21.3 \pm 11.1$ vs. $28.8 \pm 11.5$ minutes, $\mathrm{p}=0.003)$ times but less procedural complications ( $21 \%$ vs. $5 \%, p=0.042$ ) and hospitalisations during follow up ( $26 \%$ vs. $8 \%, \mathrm{p}=0.035)$. At post ablation testing, more patients were non-inducible for any VT who underwent scar homogenisation compared with the standard approach ( $69 \%$ vs. $42 \%, p=0.01$ ). During follow up (mean, $14 \pm 2$ months), patients in the scar homogenisation group had significantly more freedom from VA recurrence (64\% vs. $38.6 \%$, $\log$-rank $\mathrm{p}=0.031$ ). Whilst the results proved a benefit over a standard ablation approach, the success rates in this study were lower than those in the same ICM groups previously reported, ${ }^{18)}$ presumably due to septal and intramural scar distributions in IDCM.

\section{Arrhythmogenic cardiomyopathy}

Arrhythmogenic cardiomyopathy (AC; originally termed ARVC) is a more extensively studied NICM aetiology with CA, but results of medical therapy are not widely reported. Left ventricular involvement in $\mathrm{AC}$ is common, occurring in 30\% of patients, as is biventricular involvement, hence the term AC is more relevant than ARVC. ${ }^{21} \mathrm{CA}$ has been largely reserved for patients with high ICD burden despite AAD or beta-blocker therapy. ${ }^{22)}$ Mahida et al., ${ }^{22)}$ reported on a 109 ARVC patients retrospectively analysed from 5 international centres that received $\mathrm{AAD} /$ beta blocker therapy alone or in conjunction with a VT ablation procedure. ${ }^{22}$ Of the study group, $32(29 \%)$ received an ablation procedure and AAD/beta blocker therapy whilst the remaining $77(71 \%)$ only received AAD/beta blocker therapy. Freedom from VT after a single ablation procedure was $35 \%$ at 3 years compared to $28 \%$ in the $\mathrm{AAD} /$ beta blocker group $(\mathrm{p}=0.46)$. There were no differences in outcome based on the individual AAD/ beta blocker, whether treated with medical therapy alone or with ablation. Importantly, 43 patients in the initial AAD/beta blocker group during follow up had more VT and underwent an ablation. Therefore, a total of 77 patients underwent ablation procedures with a range of 1-7 procedures required. At 3 years follow up from the last ablation, $56 \%$ of patients were free from VT. The authors concluded that multiple procedures were necessary to maintain freedom from VT, which was consistent with prior reports, ${ }^{23)}$ and a combined endo-epicardial approach was superior to endocardial only approach.

The disease state in ARVC originates in the epicardium or subepicardial layers and progresses towards the endocardium. Hence, recent ablation advances have focussed on the benefits of a combined endo-epicardial ablation approach versus an endocardial only ablation approach. Romero et al. reported a meta-analysis of endocardial versus a combined endo-epicardial approach for the management of VT in ARVC patients. ${ }^{24)}$ Of the 9 papers included, 452 patients were identified. Patients were relatively young (mean, 42 years; range, $38-53$ years) and good LVEF (mean, 55\%). There were no significant differences between acute procedural failures ( $\mathrm{p}=0.54$ ) or acute complications $(\mathrm{p}=0.13$ ) between groups. During follow up, VA recurrence was greater in the endocardial only approach compared to the combined method ( $49.6 \%$ vs. $26.4 \%, p \leq 0.0001)$, emphasising the benefit of a combined endo-epicardial ablation approach in ARVC. 


\section{Cardiac sarcoidosis}

Sarcoidosis is an inflammatory condition characterised by non-caseating granulomas that involves multiple organs. Cardiac sarcoidosis is associated with atrioventricular block, VT, congestive heart failure and SCD. ${ }^{25)}$ Cardiac sarcoid-related VT is caused by re-entry involving confluent regions of scarring on the RV endo- and epicardium, patchy scarring of the endocardial LV affecting the basal septum, anterior wall and perivalvular regions. ${ }^{25)} \mathrm{As}$ a result, elimination of all inducible VT has been shown to be difficult due to the RV and LV involvement, intramural scarring and proximity to critical epicardial vasculature prohibiting ablation. ${ }^{25}$ )

Safety and efficacy have been reported in a recent systematic review of VT ablation in cardiac sarcoidosis patients. Papageorgiou et al., ${ }^{26)}$ identified 5 retrospective case series and a total of 83 patients. Patients presented with the median number of $3 \mathrm{VT}$ morphologies (range, 2.6-4.9) per patient and the mean tachycardia cycle length of $360 \mathrm{~ms}$ (range, 326-400 ms). All patients underwent endocardial ablation, but epicardial ablation was only performed in $18 \%$. Acute success, abolishment of all inducible VT morphologies, was achieved in $66 \%$ of patients. Procedure complications were 4.7-6.4\%. Follow up periods were reported in mean or medians and ranged from 19.6-27 months. During this follow up period, 45 (54\%) patients had recurrence of VT. Further ablation procedures were required in $26(31 \%)$ patients. Across the studies, $13 \%$ of patients required a transplantation and $8.7 \%$ of patients died during follow up. The benefit of CA in these patients, whilst moderately successful in VT freedom, importantly resulted in a reduction in arrhythmia burden by $88 \%$, underscoring its role as a treatment option.

\section{Channelopathies}

A novel area of CA is for VAs in channelopathies and for the treatment and prevention of ventricular fibrillation (VF) and normalisation of baseline electrocardiogram (ECG). Initially described for Brugada syndrome, ${ }^{27)}$ ablation that normalises the ECG have been reported in patients with early repolarisation syndromes (ERS) and idiopathic VF. ${ }^{28)}$

Pappone et al., ${ }^{29)}$ have reported the largest consecutive series of symptomatic Brugada syndrome patients treated with ablation of the right ventricle (RV) epicardium. In 135 patients with Brugada ECG patterns either spontaneously or after ajmaline infusions, a combined endocardial/epicardial approach was used to target abnormal substrate. Abnormal substrate consisted of low amplitude bipolar and unipolar voltages, abnormal local activation times and electrogram durations (>200 ms). Following ablation, an ajmaline challenge was repeated for dormant Brugada pattern ECG changes. Two groups were identified, those with typical Brugada symptoms ( $\mathrm{n}=63$ ) and VT/VF inducibility with symptoms without documented arrhythmia $(n=72)$. Substrate mapping identified low voltage areas over the right ventricular outflow tract (RVOT), which were larger in group 1 vs. group 2 ( $\leq \leq 0.001)$. Following ajmaline challenge, abnormal prolonged electrograms extended from the RVOT towards the tricuspid annulus in both groups, but group 1 showed larger regions than group 2. Following initial ablation, 78 patients showed reappearance of coved ECG patterns after ajmaline challenge and required further ablation. Further ablation eliminated any diagnostic Brugada-pattern ECG changes. Before discharge, all patients showed a normalised ECG. No procedure related complications were reported. During follow up (10 months; IQR, 8-12 months), continued ECG normalisation before and after ajmaline infusion was observed. There were no episodes of VT/VF after interrogation of ICD in $98.5 \%$ of patients.

In the largest series on ERS, Nademanee et al. ${ }^{28}$ identified 2 phenotypes; ERS with Brugada ECG patterns, and ERS without Brugada ECG patterns. Thirty-three patients with ERS and 
Brugada ECGs and 7 patients only with ERS were identified. Of these, 25 patients had VF drivers mapped by electrocardiographic imaging (ECGi). The distribution of VF drivers by ECGi and abnormal late fractionated EGMs colocalised to the same regions, predominantly in the RV epicardium (100\% correlation) of patients with ERS with or without Brugada ECG patterns. ECG was normalised post ablation in $97 \%$ of the patients with ERS and Brugada pattern, and ERS pattern disappeared post ablation in $82 \%$ of the patients. The inducibility of VF post ablation in patients with ERS decreased from $89 \%$ to $17 \%(\mathrm{p} \leq 0.001)$. After a single procedure, $67 \%$ of patients remained free of VF without AADs, which improved to $91 \%$ after a repeat procedure during mean follow up of $31 \pm 26$ months.

\section{POLYMORPHIC VENTRICULAR TACHYCARDIA/ VENTRICULAR FIBRILLATION ABLATION}

Curative treatment of polymorphic VT or VF has been focussed on ablation of the initiating beat of VF that matches the preceding premature ventricular contraction (PVC) morphology and coupling intervals. ${ }^{30-33)}$ Though once initiated VF is maintained by the continual formation of re-entrant wavefronts due to depolarisation and repolarisation heterogeneities ${ }^{34)}$ and has been shown to anchor to a scar or structural heterogeneity. ${ }^{3536)}$ Haissaguerre et al., ${ }^{30)}$ in the initial description of this approach reported up to $85 \%$ of the triggering PVCs were localised to the Purkinje conducting system and ablation of the triggers had an impressive $(89 \%)$ VF freedom without AAD over long term follow up ( $24 \pm 28$ months). In a follow-up study, Haissaguerre et al., ${ }^{33)}$ also showed that targeted PVC trigger ablation is also viable in patients with long QT syndrome (LQTS) and Brugada syndrome.

Trigger ablation frequently is a frustrating endeavor when the PVC is not spontaneous or inducible or becomes electrically quiescent. ${ }^{32 / 37}$ In one report up to $1 / 4$ of cases may not have a target PVC for ablation. ${ }^{37)}$ In these instances ablation has been reportedly guided by pace mapping, Purkinje network modification and RVOT PVC triggers electrical isolation of the pulmonary artery. ${ }^{32}$ Nakamura et al. ${ }^{377}$ recently reported on CA of polymorphic VT and VF in patients with and without SHD in 32 patients. In patients $(n=13)$ with idiopathic VF, sustained monomorphic VT was not induced but all patients had a VF triggering PVC identified and targeted for ablation. Patients with SHD $(n=19)$ had either a trigger $(n=4)$, substrate $(n=8)$ or a combination $(n=7)$ ablation approach. A low voltage region was present in $79 \%$ of patients with SHD and sustained monomorphic VT associated with scar. During follow-up (median 540 days) $74 \%$ of SHD patients and $77 \%$ of idiopathic VF patients were free of recurrence. Therefore, a reasonable approach in patients with polymorphic VT/VF and SHD is for substrate ablation in the absence of triggering PVC targets. Though patients with idiopathic VF require trigger identification and ablation.

\section{RISK ASSESSMENT FOR VENTRICULAR TACHYCARDIA ABLATION}

Pre-procedural planning engaging a multi-disciplinary team ensures the best clinical practice for the treatment of complex VT substrates. To further this approach, the development of pre-procedural assessments for a patient's acute hemodynamic compromise and mechanical circulatory support during VT ablation led to the PAINESD ${ }^{38)}$ and the I-VT score. ${ }^{39)}$ These assessments identify a patient's risk of peri-procedure acute hemodynamic decompensation 
(AHD), their need for mechanical circulatory support, and predict their survival free from VT recurrence, early mortality (<30 days), and 1-year mortality following VT ablation.

The PAINESD score is a risk score that utilises the following characteristics: chronic obstructive pulmonary disease, age $>60$ years, ICM, NYHA $\geq$ class 3 , LVEF $<25 \%$, VT storm and diabetes mellitus to generate a risk profile score. Each variable is given a weighted value and a total score classifies patients as low (score $\leq 8$ ), intermediate (score 9-14) or high-risk (score $\geq 15$ ). ${ }^{40)}$ The risk of periprocedural AHD is estimated to be $1 \%, 6 \%$ and $24 \%$ in low, intermediate and highrisk groups ${ }^{40)}$ Subsequently if rescue support is required, the odds of 30 -day mortality post VT ablation is $58 \%$ without rescue support and $3 \%$ with rescue support. ${ }^{40)}$

Vergara et al. ${ }^{39)}$ created an assessment of a patient,s risk of VT-free survival and mortality by calculating the I-VT score by survival tree analysis in 1,251 patients across 16 demographic, clinical, and procedure-related variables. The I-VT score, expressed as a HR, comprises of pre- and post-procedural characteristics. Pre-procedure these comprise of clinical characteristics of age, LVEF, presence of VT storm, presence of an ICD or cardiac resynchronisation therapy device (CRT), ICM and NICM, and prior VT ablation procedure. Post-procedure the I-VT score included diabetes and results of programmed stimulation at the end of the ablation procedure. Vergara et al. reported three distinct groups; high, medium, and low risk. Survival in the high-risk group was $65 \%$ and $52 \%$ were free from VT recurrence. Low risk patients in contrast had a $97 \%$ survival and an $88 \%$ freedom from VT recurrence rate. Preoperative risk stratification may assist in procedural planning but utilisation in prospective trials needs to be completed for confirmation of long-term outcomes. Post-procedure risk scores may also help in family counselling and planning. ${ }^{39)}$

\section{CARDIAC IMAGING}

Pre-procedural image integration by computed tomography (CT) or cardiac magnetic resonance (cMR) imaging into EAM systems has been available since the early 2000s. ${ }^{\left.41)^{42}\right)}$ Initially for pure anatomic navigation recent advances have focussed on tissue characteristics by late gadolinium enhancement cMR (LGE)-cMR or contrast enhanced perfusion multidetector computed tomography (CEP-MDCT) ${ }^{43-46)}$ The assessment of tissue characteristics has guided VT ablation of conducting channels and assessments of lesion efficacy. ${ }^{47)}$ The pattern of LGE-cMR distribution has also been shown to correlate with scar patterns of NICM. ${ }^{48)}$ Two typical scar patterns have been reported; anteroseptal and inferolateral. These typical distributions were associated with 3 distinct VT morphologies; right bundle branch block, inferior axis and positive concordance; left bundle branch block, inferior axis, and early $(<\mathrm{V} 3)$ precordial transition; right bundle branch block, right inferior/superior axis and late $(>5)$ precordial transition. Wall thickness by CEP-MDCT has been shown to be critical in post infarction VT substrate identification. ${ }^{49) 50}$ Severe wall thinning $(<2 \mathrm{~mm})$ regions frequently harboured complex signals critical for VT $(91 \%)$ and were found in ICM and post myocarditis patients but not commonly in IDCM.

\section{Image processing of tissue characteristics}

Two commercially available software platforms have become widely accepted for processing pre-procedural imaging scans whether done by LGE-cMR or CEP-MDCT; ADAS-VT (Galgo Medical SL, Barcelona, Spain) and MUSIC (Liryc-Université de Bordeaux/Inria-Sophia Antipolis, France). ADAS-VT utilizes the 3-dimensional (3D) distribution of pixel signal 


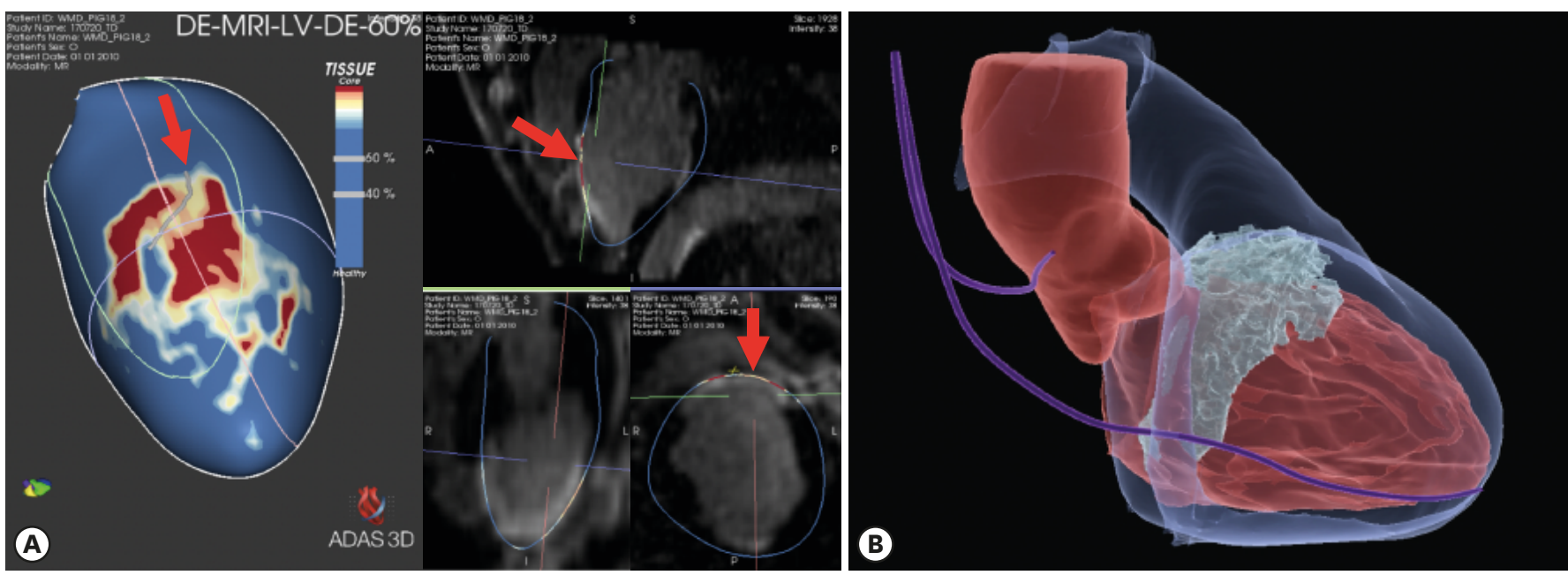

Figure 1. Preprocedural imaging of scar. (A) LGE-MRI 3-dimensional reconstruction of the LV from a porcine infarct model with ADAS-VT. To delineate tissue characteristics PSI set to $60 \% / 40 \%$. Red region core scar location and blue is healthy tissue. Reconstruction of LV with small tube denoting conducting channel identified by ADAS (Red arrow). MRI images of associated location of conducting channel. (B) Music images of LV and RV in EAM system. White structure is scar reconstruction at the peri-aortic and basal septal region. ICD leads shown in purple. Music images courtesy of Dr. Saagar Mahida, Liverpool Heart and Chest Hospital, Liverpool, UK.

EAM = electro-anatomic mapping; ICD = implantable cardioverting defibrillator; LGE = late gadolinium enhancement; $L V=$ left ventricle; $M R I$ = magnetic resonance imaging; $\mathrm{PSI}=$ pixel signal intensity; RV = right ventricle; VT = ventricular tachycardia.

intensity variations from pre-procedural imaging to characterize the myocardial fibrosis and project the intensities of the fibrosis on to layers of the myocardial wall (Figure 1). MUSIC enables the fusion of multi-modality imaging sequences (CT or CMR) into a single dataset and then the creation of 3D volume files for integration to the EAM are generated.

Using ADAS-VT, and a 40-60\% pixel signal intensity threshold with LGE-cMR, investigators were able to locate dense and border zone regions of fibrosis and identify conducting channel exits/entrances in patients with SHD. ${ }^{51)}$ Ablation targeting the conduction channels identified using ADAS (termed "de-channeling") resulted in the reduced need for RF delivery and high rates of non-inducibility compared to patients with a non-cMR guided ablation procedure. ${ }^{52}$

A current limitation of cMR imaging in these patient groups is the presence of ICD leads causing significant artifact and limiting the image quality required for interpretation of scar. Roca-Luque et al. ${ }^{53)}$ reported the use of a wideband LGE-cMR sequence in 13 patients with ICDs for VT substrate characterization and definition of conducting channels. Patients were matched to conventionally scanned LGE-cMR patients before ICD implantation and VT ablation and conduction channels confirmed by EAM. There was no significant difference $(\mathrm{p}=0.19)$ of the accuracy of conducting channels identified with wideband sequence vs. EAM $85.1 \%$ vs. $92.2 \%$, false positive detection in cMR to EAM in either wideband or conventional (7.5\% vs. $7.8 \%, p=0.9$ ). The authors concluding that wideband LGE-cMR could guide VT ablation using ADAS with similar accuracy as compared to conventional LGE-cMR in patients without an ICD.

The influence of scar identified with ADAS has been explored as a predictor for the response to PVC ablation in patients with LV dysfunction. Penela et al., ${ }^{54)}$ reported the results of 70 patients with reduced LV function (LVEF $<50 \%$ ) from 4 centers who received LGE-cMR scan prior to PVC ablation. Myocardial scar was identified in $29(41 \%)$ patients and PVC site of origin was located predominantly in the LV (73\%) than RV (27\%). Acute success was similar 
between patients with and without scar ( $88 \%$ vs. $83 \%, p=0.4$ ). PVC burden was reduced after ablation from $23 \%(18-32)$ at baseline to $1 \%(0-7)$ at 12 months $(\mathrm{P} \leq 0.001)$. Patients were considered echocardiographic responders if LVEF improved by $5 \%$ during follow up and was achieved by $69 \%$ of patients. Non-responders had significantly larger scar mass than responders (2 [0-14] vs. 0 [0-4.7] grams, $\mathrm{p}=0.017)$.

Whilst LGE-cMR is becoming the gold standard of tissue characterization, ${ }^{49}$ as mentioned previously there are limitations of cMR sequences in patients with ICDs which have led to further investigation of CEP-MDCT. CEP-MDCT is generalizable to clinical practice and is a validated modality for post-myocardial infarction scar identification. ${ }^{5556)}$ Ustunkaya et al., ${ }^{45}$ evaluated 14 patients with post infarction VT for the association of wall thickness and signal attenuation on CEP-MDCT with conduction velocities and electrogram abnormalities. Myocardial hypoattenuation on CEP-MDCT was associated with reduced conduction velocity and decreased bipolar and unipolar EGM amplitudes. These hypo-attenuated regions were more likely to harbor EGMs of increased duration, fragmentation, and late components. Ustunkaya et al. ${ }^{46)}$ further described CEP-MDCT utility in ARVC patients for conduction velocities and electrogram abnormalities. $\mathrm{RV}$ attenuation was associated with bipolar epicardial voltage amplitude, unipolar endocardial amplitude, and with epicardial conduction velocity. Esposito et al. ${ }^{56)}$ reported delayed enhancement CT scans with hyper attenuation and its relation to EAM substrate in 42 patients. The majority of patients (83\%) had an ICD at the time of scan. The CT scans identified scars in 39 patients and defined the LV wall involvement and the mural distribution. CT identified segments correlated with low voltage EAM regions with good sensitivity ( $76 \%$ ), specificity ( $86 \%$ ) and a high negative predictive value $(95 \%)$. Additionally, late potentials and sites of RFA localized to scarred CT segments in $79 \%$ and $81 \%$ of cases. Notably, there is a relatively low contrast to noise ratio when compared to LGE-cMR with hyper-attenuation, which may impact the ability of delayed enhanced CT to identify dense core scar and border zone regions where conducting channels are most frequently found.

\section{ELECTROPHYSIOLOGICAL MAPPING}

Advancements in EAM have focused on the utilisation of high density electrogram sampling throughout the chamber of interest and differing techniques for interpretation of the data. The functional assessment of VT substrate has also been explored.

\section{Omnipolar mapping}

The Omnipolar method was first described by Deno et al. ${ }^{57)}$ and validated by Masse et al. ${ }^{58)}$ in an animal model. An omnipole was described as resolving a signal from all possible simultaneous bipoles (from every direction around the mapping location) from the electric field generated by a travelling wave for the definitive assessment of local cardiac wavefront properties, such as speed and direction. ${ }^{57 / 58)}$ An omnipolar voltage was further described for the definition of ventricular and atrial substrate, defining voltages along the maximal bipolar direction of propagation, ${ }^{59-61)}$ using a similar methodology. Omnipolar voltages in a porcine infarct model were shown to be larger than traditional bipoles, more consistent when in the infarcted area, and overall substrate maps better correlated with scar defined by cMR.

Porta-Sanchez et al., ${ }^{61)}$ compared omnipolar-derived bipolar EGMs with conventional bipolar EGMs from the HD grid (HDG) catheter (Abbott Medical, Abbott Park, IL, USA) in an animal 
infarct model. The omnipolar derived EGMs vs. conventionally derived bipolar EGMs had a $26 \%$ and $23 \%$ larger value for healthy and infarcted zones respectively and a better correlation to the LGE-cMR infarction zones, minimising the overestimation of conventional bipolar substrate maps.

\section{Isochronal late activation mapping}

Isochronal late activation mapping (ILAM) aims to identify not the latest activation but the entry sites into the latest regions of ventricular activation. Irie et al., ${ }^{62}$ initially described ILAMs in a retrospective study of 33 patients with ICM and prospectively in 10 patients. Electrogram timings were manually annotated to the offset of the local bipolar deflection, signifying completion of local activation during sinus rhythm. Activation timing was displayed as isochrones, separated into 8 equally distributed time periods of $12.5 \%$ of ventricular conduction (Figure 2). ILAMs were then identified as deceleration zones (DZ) by $>2$ isochrones crowding within a $1 \mathrm{~cm}$ radius. ILAM sites were then compared with critical sites of termination with ablation in 47 VTs. At termination sites, the median ventricular activation percentage was $78 \%$ and $18 \mathrm{~mm}$ removed from the centre of the latest isochrone. Prospectively the regions of second $(75-87.5 \%)$ and third latest (62.5-75\%) that bunched outside of the latest isochrones were targeted for ablation. Acutely $40 \%$ of patients were successful and $50 \%$ partially successful. Mid-term follow up at 6 months showed $80 \%$ of patients were free from VT.

In a large series, Aziz et al., ${ }^{63)}$ prospectively enrolled 120 patients with scar-related VT (ICM $50 \%$, NICM 50\%) in a registry. Targeted ablation was performed at DZ ( $>3$ isochrones within $1 \mathrm{~cm}$ radius), prioritizing later activated regions with maximal isochronal crowding. $\mathrm{DZ}$ were located in mixed scar $(<1.5 \mathrm{mV})$ tissue in $35 \%$ and in dense scar $(<0.5 \mathrm{mV})$ in $63 \%$, with $2 \%$ of $\mathrm{DZ}$ in normal voltage $(>1.5 \mathrm{mV})$. The mean number of DZ identified was $2 \pm 1(18 \%$ had a single $\mathrm{DZ}$, 35\% with $2 \mathrm{DZ}$, and $45 \%$ with $>3$ ). DZ were correlated to optimal pace mapping sites $(92 \%)$ and entrainment with concealed fusion $(63 \%)$, and colocalised to the VT termination site (95\%). At $12 \pm 10$ months follow up VT freedom was $70 \%$ but patients with ICM tended to have improved freedom from VT compared to NICM patients ( $80 \%$ vs. $63 \%, \mathrm{p}=0.094$ ).

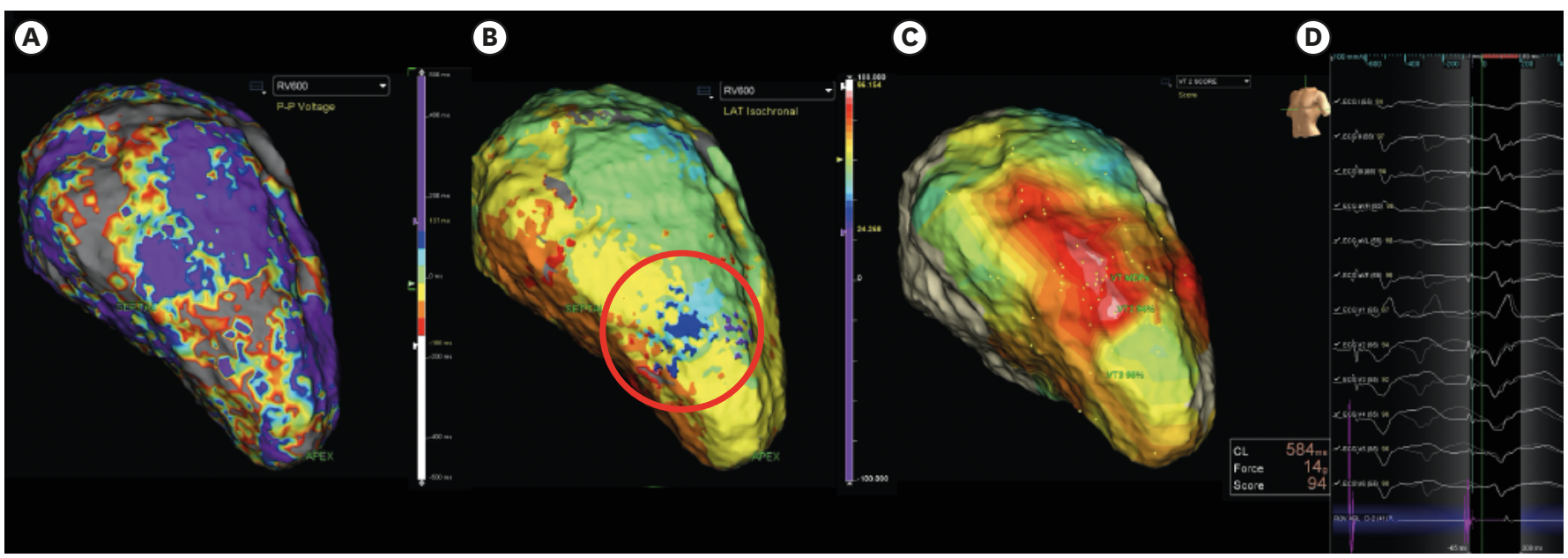

Figure 2. ILAM and pace mapping correlation map. (A) LV EAM reconstruction of substrate voltage in RVp (Bipolar 0.5-1.5mV). (B) LV EAM reconstruction of activation time in RVp. ILAM displayed at red circle. Note rapid change in colors denoting slow conduction over a small area. (C) Pace map correlation for VT. Best pace map for VT (white region) localizes to same regions as ILAM and low voltage in substrate mapping. (D) Pace mapping signals from ILAM region shows $94 \%$ match to VT morphology with 80 ms stimulation to QRS delay. EAM = electro-anatomic mapping; ILAM = isochronal late activation mapping; LV = left ventricle; RVp = right ventricular pacing, $V T=$ ventricular tachycardia. 
Furthermore, ablation times were significantly lower than techniques that employed complete substrate homogenisation. Notably, the advantage of this technique is limiting the amount of ablation to achieve VT non-inducibility and at least equivalent outcomes to more extensive scar homogenisation where extensive RF may be necessary, resulting in longer procedure times. ${ }^{63}$

\section{Ripple mapping}

A common limitation of activation and substrate mapping is signal annotation on complex multi-component electrograms. Cardiac ripple mapping was first developed by Linton et al. in $2009,{ }^{64)}$ to dynamically display activation and voltage amplitude simultaneously for cardiac arrhythmias independent of annotations. (Figure 3) Jamil-Copley et al. ${ }^{65)}$ retrospectively analysed 21 patients with post-infarction VT, showing functional conduction visualised with ripple mapping occurred through low voltage regions using standard bipolar voltage cut offs (0.5-1.5mV) during substrate mapping. During follow up (median 466 days) $71 \%$ of patients remained VT free. This study identified that a universal low-voltage cut off is unsatisfactory and voltage thresholds need to be more patient specific.

Luther et al. ${ }^{66)}$ in a prospective non-randomised feasibility study used ripple mapping to identify conduction channels through low voltage regions in 15 patients with post-infarction VT. Focussed ablation limited to the conduction channels resulted in $85 \%$ being non-inducible for VT and in 70\% freedom from VT at 6 months. Larger studies are required to confirm if targeted ablation of ripple mapping identified conduction channels will improve outcomes.
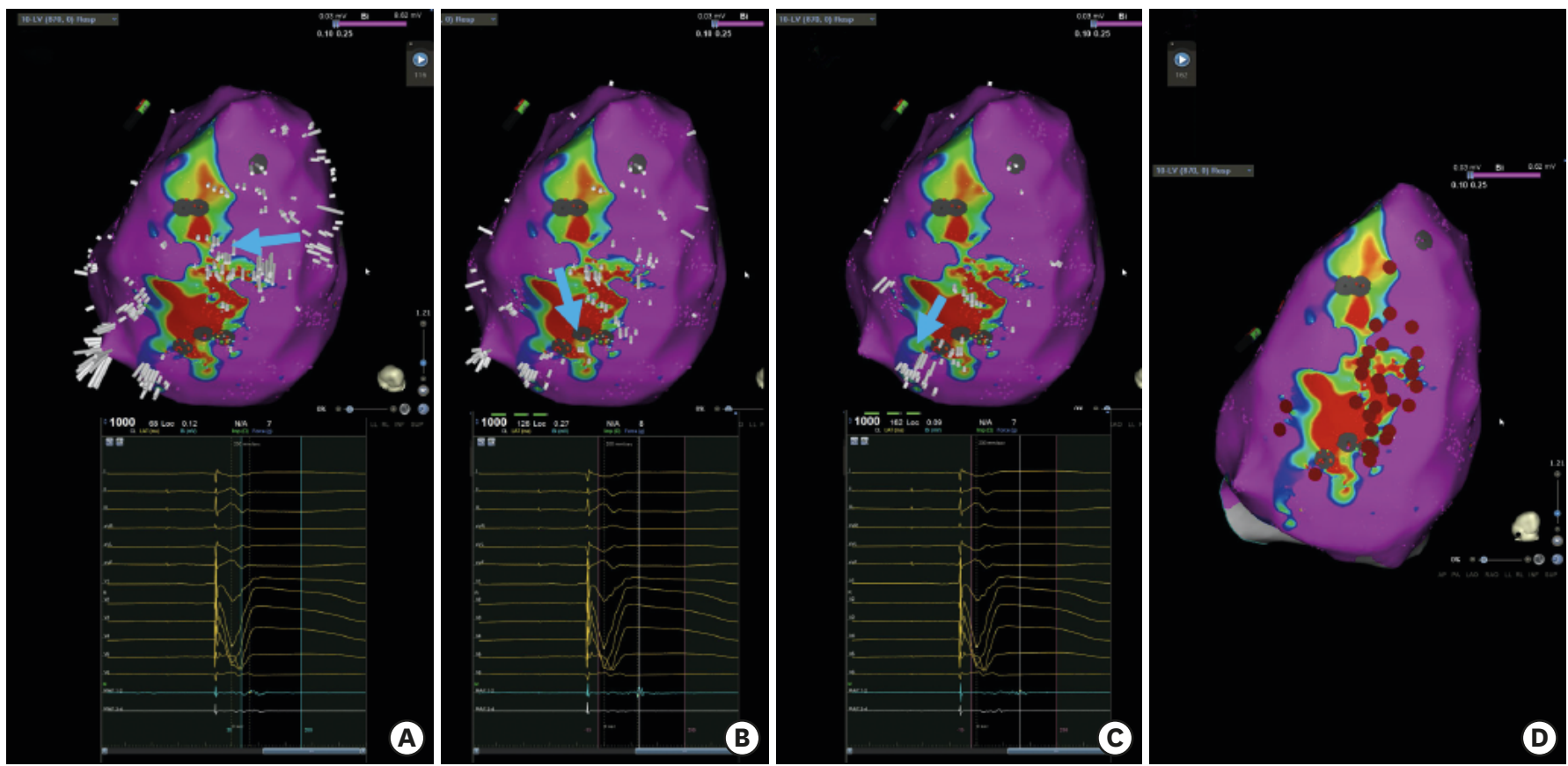

Figure 3. Conducting channels on ripple mapping in inferior view of LV. Blue arrow denotes course of conducting channels identified with Ripple mapping. (A) EAM reconstruction of voltage map with Ripple bars displayed and associated EGM at border zone. Voltages cutoff values are reduced (0.1-0.25 mV) to show viable tissue below standard cutoffs. Ripple bars at entrance point to conducting channel. (B) Ripple bars show conducting channel propagation through low voltage from mid to annular region of scar and associated EGMs of this region. (C) Termination of conducting channel at annular region. EGMs show latest activation of conducting channel. (D) Ablation that rendered the patient non-inducible for all VT at completion of procedure. Ablation tags in red. EAM = electro-anatomic mapping; EGM = electrogram; LV = left ventricle; VT = ventricular tachycardia. 


\section{Decrement evoked potential mapping}

Conduction delay and unidirectional block are requirements for re-entry. Identifying these regions with traditional substrate maps is limited as the substrate is relatively fixed in a consistent rhythm used for mapping. Decrement evoked potential (DEEP) mapping was developed to bridge the gap in localising these regions using functional electrophysiologic techniques. DEEP mapping utilises a single extra-stimulus that delays timing in near field potentials. ${ }^{67}$ Annotation of the latest sharp, near-field component of bipolar electrograms during the pacing train $(600 \mathrm{~ms})$ and on the first extra-stimulus (ventricular effective refractory period $+20 \mathrm{~ms}$ ) was compared at sites of late potentials or fractionation. ${ }^{67}$ Differences $>10 \mathrm{~ms}$ were categorised as DEEP and timing windows were manually set to show sites of delay ${ }^{67}$ DEEP mapping was validated with simultaneous global intraoperative mapping, and found potentials with decremental properties within scar or border zones of scar identify sites participating in VT circuits (specificity $50 \pm 23 \%$ and sensitivity $43 \pm 23 \%$ ). In a prospective multi-centre observational study, Porta-Sánchez et al. ${ }^{68}$ used DEEP mapping in 20 patients with ICM to deliver focussed ablation as a strategy to reduce VT burden. In each patient sites of late potentials were assessed for decremental conduction. Sites that decremented were defined as DEEP and late potentials that did not decrement were defined as non-DEEP-LP. Regions of DEEPs were localised more frequently in the diastolic pathway of VT than non-DEEP-LPs (specificity, 0.97 [95\% CI, 0.95-0.98] vs. 0.82 [95\% CI, 0.73-0.89]) and were identified in smaller areas of the mapped myocardium than non-DEEP-LPs $(4.8 \%$ [IQR, $2.2-25.7 \%$ ] vs. $16.8 \%$ [IQR, 8.9-73.7\%], $\mathrm{p}<0.001$ ). Acutely $80 \%$ of patients were non-inducible after DEEP focused ablation and $75 \%$ were VT free at 6 months follow up. VT burden was significantly reduced for both amount of VT (11 [IQR, 5-25] vs. 0 [IQR, 0-2], $\mathrm{p}=0.02)$ and number of shocks from ICD (1.5 [IQR, 0-4.5] vs. 0 [IQR, $0-0], \mathrm{p}=0.03)$ post ablation. The feasibility of targeting the functional regions responsible for re-entry in postinfarct VT is encouraging. The applicability of DEEP mapping in NICM where attempting to elicit a decremental uncoupling of electrograms and intramural substrates requires further investigation.

\section{Electrocardiographic imaging}

The inverse solution of the electrocardiogram is a tantalising prospect for electrophysiologists for non-invasive localisation of VAs. The first commercially available system (CardioInsight, Medtronic, Minneapolis, MN, USA) to provide ECGi provides panoramic mapping over one cardiac cycle by non-invasive reconstruction of epicardial unipolar electrograms using heart-torso geometry generated by CT or MRI and body surface potentials. ${ }^{69}$ It has been reported to localise focal and macro re-entrant arrhythmias and provide substrate identification correlated with LGE MRI scans ${ }^{69) 70)}$ ECGi has recently been utilised for the study of VF ablation in channelopathies ${ }^{2877173)}$ were the results were encouraging, as discussed in the previous channelopathies section.

Recently, Graham et al. ${ }^{74)}$ compared the accuracy of ECGi with simultaneous invasive mapping via an EAM system in 37 patients with structurally abnormal heart VT. In eighteen patients included for analysis sites of origin of earliest activations of VT by ECGi and EAM were a median $22.6 \mathrm{~mm}\left(\mathrm{IQR}_{25-75 \%}, 13.9-36.2 \mathrm{~mm}\right)$ apart. Non-significant differences were observed in ICM and NICM patients (median, $26.6\left[\mathrm{IQR}_{25-75 \%}\right.$, 18.5-39.1 mm] vs. $15.8\left[\mathrm{IQR}_{25-}\right.$ $75 \%$ [9.6-21.6 mm], $\mathrm{p}=0.055)$. The authors concluded the current iteration of ECGi lacks adequate accuracy and precision to guide CA without detailed invasive contact mapping, but as the accompanying editorial by Sapp et al. also noted this may be enough for novel methods of therapy delivery like stereotactic body radiation therapy (SBRT). ${ }^{75)}$ 


\section{ADVANCES IN MAPPING CATHETERS}

Advances in catheter design have centred on multi-electrode mapping (MEM) catheters that improve density, resolution, and speed of mapping. ${ }^{76)}$ This has coincided with advances in EAM systems capable of processing rapid amounts of sampled data. Potential limitations of MEM catheters include mechanical trauma with frequent ectopy, transient injury of the superficial conduction systems, limited catheter manipulation, lack of tissue contact, and need for anticoagulation to reduce risk of thrombus formation. ${ }^{76)}$

\section{HD grid}

The HDG is an asymmetric deflecting, irrigated 16-electrode MEM catheter arranged in a four-by-four electrode configuration with an interelectrode distance of $3 \mathrm{~mm}$ edge-to-edge of $1 \mathrm{~mm}$ electrodes. ${ }^{77)}$ Four splines are orientated parallel to each other with electrodes along the splines. Electrodes on an adjacent spline are equally spaced. Irrigation ports are located at the junction of the distal main catheter body and the HDG splines and irrigated via a pressure bag with heparinised saline at a minimum of $2 \mathrm{~mL} / \mathrm{min}$. Bipolar electrograms can be created in a linear or across spline configuration to enable bipolar EGM creation at orthogonal orientations. The HD Wave solution ${ }^{\mathrm{TM}}$ (Abbott Medical) combines the HDG catheters' ability to collect orthogonal electrograms from the same location and the best duplicate algorithm $(\mathrm{BDA}){ }^{78)}$ The BDA selects the EGM with the best-combined timing and voltage, favouring those EGMs with large voltages that are close to average timing. ${ }^{.9)}$ Duplicate EGMs are stored in the system and can be assessed to ascertain the amount of compared EGMs per single site. Okubo et al..$^{78}$ recently published the HDG catheter and BDA use in identifying late potentials and diastolic isthmuses in a SHD population with VT. The HD wave solution generated substrate maps showed a significantly smaller low voltage area in comparison to linear and across spline EGM configurations. In an animal infarct model, Takigawa et al. ${ }^{80}$ demonstrated using the BDA provided a higher accuracy for scar discrimination compared to not using the algorithm regardless of EGM collection configuration; along, across or diagonal between electrodes. Recently Campbell et al. ${ }^{77)}$ showed that the BDA software when used with the HDG catheter resulted in only $9 \%$ of acquired EGMs being used. Whilst the catheter is safe and feasible for use in VAs there becomes a diminishing return on further collection of EGMs during mapping.

\section{Octaray}

The Octaray ${ }^{\mathrm{TM}}$ (Biosense Webster, Inc., Irvine, CA, USA) is a new iteration of the Pentaray ${ }^{\mathrm{TM}}$ catheter currently in pre-clinical studies. ${ }^{81)(22)}$ The Octaray has been developed to incorporate 8 splines with 6 electrodes per spline in a $2 \mathrm{~mm}$ edge to edge spacing configuration. Electrode surface area size has been reduced compared to the traditional Pentaray catheter ( 0.9 vs. 2 $\mathrm{mm}^{2}$ ). In a direct comparison of Pentaray and Octaray catheters, Barkagan et al., ${ }^{81}$ showed in an animal infarct model the novel catheter increased mapping speed, EGM density and an improved ability to identify low amplitude EGMs in infarcted myocardium.

\section{Orion}

The era of ultra-high-density mapping was instigated by the Orion ${ }^{\mathrm{TM}}$ catheter and Rhythmia ${ }^{\mathrm{TM}}$ mapping system (Boston Scientific, Marlborough, MA, USA). Viswanathan et al. ${ }^{83)}$ reported their initial evaluation of the Orion ${ }^{\mathrm{TM}}$ 64-electrode mini-basket mapping catheter and EAM (Rhythmia $^{\mathrm{TM}}$, Boston Scientific) for VAs in a retrospective study across 2 centres. Total substrate mapping and activation times were reported for 20 patients, 24 minutes (14:5934:22 minutes) and 9 minutes (04:12-16:14 minutes), respectively. Liu et al., ${ }^{84}$ evaluated the 
same catheter in predominantly right sided outflow tract procedures and showed reduced mapping and ablation times. The mapped rhythms were predominantly activation mapped and did not necessitate an additional substrate map.

\section{Mini electrodes integrated to ablation catheter electrodes}

Resolution of ablation catheter EGMs is often poor due to the required size of an ablation electrode for effective current delivery during RFA. An approach to improve ablation catheter signal resolution is to integrate mini electrodes which have been reported previously. ${ }^{85)}$ Recently the application of local impedance measurements through a similar catheter (IntellaNav MIFI OI ${ }^{\mathrm{TM}}$, Boston Scientific) for improved tissue characterisation in patients with VT has been described. ${ }^{86)}$ Prospectively, 28 patients with recurrent VT, 64\% ICM, 25\% NICM and $11 \%$ idiopathic were enrolled. Local impedance (LI) as measured through catheter tip to a proximal ring electrode showed improved differentiation of scarred to healthy myocardium (scarred 87.0 $\Omega$ [IQR, 79.0-95.0] vs. healthy $97.5 \Omega$ [IQR, 82.75-111.50]; $\mathrm{p}=0.03$ ). Whereas global impedance (GI) measured from tip to return electrode on the patient's skin did not show any differences between the 2 regions (scarred $109 \Omega$ [IQR, 100.0-115.0] vs. healthy 107 $\Omega$ [IQR, 101.0-107.0]; $\mathrm{p}=0.51$.

A separate ablation catheter with microelectrodes has also been reported in a pre-clinical study for mapping of ventricular scar (Qdot, Thermocool Smarttouch SF-5D Catheter, Biosense Webster, Inc.). ${ }^{87}$ Electrode sizes ( 1 vs. up to $0.15 \mathrm{~mm}$ ) and position of the small electrodes are different between Intellatip OI MIFI and the Qdot. Micro-electrodes identified preserved sub-endocardium channels more accurately than standard ablation electrodes when compared to LGE-cMR..$^{87}$ In a separate pre-clinical study, Glashan et al. ${ }^{88}$ confirmed the findings with transmural biopsies and direct histological analysis of conventional and microelectrodes integrated to an EAM system. Additionally, they identified cut-off values for use with the Qdot catheter for conventional unipolar $(<5.44 \mathrm{mV})$, bipolar $(<1.27 \mathrm{mV})$ and micro-electrode $(<2.84$ $\mathrm{mV}$ ) low voltages. Individualised substrate identification ranges are becoming an increasingly important requirement for all catheters being used for substrate mapping.

\section{ADVANCES IN ABLATION}

Recent advances in ablation technology for VT have centred on the delivery of radiofrequency (RF) energy more efficiently to the myocardium, novel irrigants to change the ohmic resistance of the ablation circuit, and therapeutic options for regions previously unreachable by current techniques.

\section{Contact force}

Contact force (CF) sensing catheters have become standards of care in recent years. CF effective ranges for ablation are region specific depending on the route of access to the

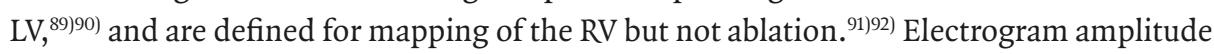
and frequency of late potential identification has also been shown to be determined by the amount of CF. ${ }^{92}$

Optimal CF ranges for the effectiveness of ablation have been reported recently by Elsokkari et al. ${ }^{90)}$ in ischemic scar-related VT procedures. Twenty-five procedures in 24 patients from a single centre were analysed. Effective lesion formation was defined as electrical un-excitability immediately post ablation at sites which were electrically excitable prior to 
ablation with unipolar pacing stimulus (10 $\mathrm{mA}$ and $2 \mathrm{~ms}$ pulse width). $\mathrm{LV}$ access was obtained from retrograde aortic in 18 , and transseptal in 7 procedures. Within the scar zone, mean $\mathrm{CF} \geq 10$ g was significantly associated with effective lesion formation (OR, 3.21 [95\% CI, 1.43-7.19], $\mathrm{p}=0.005$ ). Inducibility testing post ablation was only performed in 15 procedures and the remainder it was not performed due to length of procedures or to not perform further ablation. Of the patients tested 12 of the $15(80 \%)$ had no inducible VT. Using a 4 segment model of the LV, the anterior segment had a higher median CF with retrograde aortic (28 vs. $16 \mathrm{~g}, \mathrm{p}=0.004$ ) but transseptal was higher in the lateral segment ( $21 \mathrm{vs.} 18 \mathrm{~g}, \mathrm{p}=0.032)$ in contrast to previous reports. ${ }^{89}$

Etiology specific CF usage has been primarily reported in ICM or mixed SHD patients. Aras et al. recently reported on CF usage in 17 patients with diagnostic task force criteria for arrhythmogenic right ventricular cardiomyopathy. ${ }^{93)}$ Groups were analyzed between PVC ( $\mathrm{n}=5$ ) and VT $(\mathrm{n}=12)$. Of note, the VT patients were all presenting as VT storm. Mean endocardial CF during mapping and ablation was $12.6 \pm 1.1$ and $12.8 \pm 1$.9 grams in the PVC group and 12.9 \pm 1.1 and $12.8 \pm 1.9$ grams in the VT group. The VT group also had epicardial mapping and ablation, where the mean mapping and ablation were $14.4 \pm 2.6$ and $12.1 \pm 1.4$ grams. Acutely in 2 patients, all morphologies could not be abolished. During follow up (mean, $15 \pm 3.1$ months) PVC burden was reportedly reduced and all patients had improvements in LVEF. Acutely all patients in the VT group had successful abolishment of clinical VT, and 9 of the 12 (75\%) complete success of all VTs. In the VT group, none of the patients represented with VT storm and three patients had appropriate ICD therapies during the mean follow up of $15.5 \pm 4.5$ months.

Outcomes related to CF sensing catheter use in VA ablations have been shown to not improve procedural outcomes or safety profiles in comparison to non-CF sensing catheters in observational studies. ${ }^{94)}$ Recently Elbatran et al. ${ }^{95)}$ in a propensity-matched analysis compared acute and long-term outcomes of a 56-hole ablation tip with (Thermocool SmartTouch Surround Flow; TC-STSF) and without (Thermocool SmartTouch Surround Flow; TC-SF) CF sensing in 328 patients. Patients were matched by SHD and arrhythmia type. There was no difference between procedures with and without $\mathrm{CF}$ in acute success $(86 \%$ vs. $82 \%, p=0.3$ ), complications ( $6.7 \%$ vs. $6.7 \%, p=1.0$ ) and VA free survival (TC-STSF: mean arrhythmia-free survival time, 3.2 years; 95\% CI, 3-3.5 vs. TC-SF: mean arrhythmia-free survival time, 5.9 years; $95 \% \mathrm{CI}, 5.4-6.4$, log-rank $\mathrm{p}=0.74)$. Only fluoroscopy time (minutes) was significantly reduced in normal heart VA ablations with CF (19 [IQR, 14-30], vs. 14 [IQR, 8-25], $\mathrm{p}=0.04)$. In a separate study, de Vries et al. ${ }^{96)}$ utilised a prospective registry to specifically compare procedural and long-term outcomes of $\mathrm{CA}$ in 73 patients with idiopathic outflow tract VAs between CF sensing, no-CF sensing and a remote magnetic navigation system (RMNS). No difference in procedural success rates was observed (no-CF sensing $81 \%$, CF sensing $71 \%$, and RMNS 92\%, $\mathrm{p}=0.20$ ). However median fluoroscopy time (minutes) was reduced in RMNS when compared with the combined grouping of CF sensing and no-CF sensing (RMNS 13 [10-20], no-CF sensing 29 [16-38] and CF sensing 37 [21-46], $\mathrm{p}=0.001$ ). The role of CF sensing in VA ablation, therefore, is currently inconclusive owing to the lack of a randomised trial, though clinical intuition suggests that CF sensing catheters should make procedures faster, safer, and perhaps more effective. Further work is needed to optimise CF parameters in delivering safe and effective lesions.

\section{Novel irrigants in open irrigation ablation catheters}

Saline irrigated ablation catheters are widely used in clinical practice and enable low electrode-tissue interface temperatures during RF ablation for deeper and larger lesions 
whilst providing an improvement in safety profiles compared with non-irrigated ablation. ${ }^{97)}$ Whilst lesions are larger with saline irrigated ablation, regions critical for VT circuit interruption or focal initiating sites of VT may not be reached if deep within myocardium or substrate is complex with current approaches. Recent reports showed that low-ionic, high impedance irrigants (half normal saline [HNS]) utilising currently available open irrigated catheters improved lesion depth $10-12 \%$ and lesion volume $25-30 \% .{ }^{98) 999}$

Nguyen et al., ${ }^{100)}$ have reported a prospective, multi-centre study for the treatment of VAs refractory to standard ablation and/or bipolar ablation with HNS irrigated ablation (HNS RFA) in 78 patients. Bipolar ablation was attempted in $17 \%$ of patients before swapping intraprocedurally to HNS RFA. More than $50 \%$ of cases where HNS RFA was delivered involved the IVS or the LV summit. The remaining cases involved papillary muscles (13\%), LV free wall $(15 \%)$ and the RV $(16 \%)$. Acute success was achieved in 83\%, and 1-year VA free survival was $89 \%$. Patients without acute success predominantly had NICM substrate or LV summit focus. Complications reported were not related to HNS RFA delivery, though 12 cases reported steam pops and 1 pericarditis following epicardial HNS RFA.

In a prospective observational study, HNS RFA has been applied to outflow tract VAs following failed standard ablation techniques. ${ }^{101)}$ Thirty-one patients underwent HNS RF after standard ablation of $>300$ seconds at the best activation and/or pace mapping site, or adjacent structures failed to eliminate the VA. Successful site in the standard ablation group was predominantly in the RVOT (76.0\%), the aortic sinus of Valsalva (ASV; $10.9 \%$ ), the left ventricular outflow tract (LVOT; 7.0\%), pulmonary artery (PA; 4.7\%) and great cardiac vein/ anterior interventricular vein (GCV/AIV; $0.8 \%$ ), and intramural ( $0.8 \%)$. Whereas in the HNS group, the successful site was distributed evenly, with $38.7 \%$ success at the RVOT, $25.8 \%$ at the LVOT, $22.6 \%$ at the ASV and $6.5 \%$ at the GCV/AIV, and 6.5\% intramurally. HNS RF achieved acute success in $68 \%$ of patients, but $26 \%$ of patients at mid-term follow up (mean $7.8 \pm 4.6$ months) had recurrences, and PVC burden was significantly higher compared to the standard ablation group $(22.8 \pm 14.9 \%$ vs. $5.9 \pm 8.3 \%$; $\mathrm{p}=0.038)$. The anatomical challenges of the HNS group may explain reduced outcomes.

\section{Needle ablation}

Needle infusion ablation (NIA) has been developed as an alternative option for standard RF ablation techniques of arrhythmogenic substrate deep in the myocardium. ${ }^{1021103)} \mathrm{RF}$ energy can be delivered through a heparinised saline irrigated 27-g needle and extended for up to 10 $\mathrm{mm}$ from the dome electrode of an ablation catheter. ${ }^{104)}$

In a prospective multi-centre study, 42 patients with episodes of monomorphic VT or incessant VT and a reduced left ventricular ejection fraction $(<40 \%)$ that had failed to respond to AADs, and failed CA were enrolled for NIA. NIA in 11 patients was not performed due to endocardial arrhythmia origin in 10 patients and in 1 a large thrombus in the RV. As NIA can spare the myocardial surface, a conventional ablation catheter was also used in 14 patients. The arrhythmia target was monomorphic VT in 26 patients and frequent PVCs associated with reduced ventricular function in 5 patients. A median of 15 NIA applications was delivered per patient (range, 5-66). Acute success in the monomorphic VT group was achieved in 15 patients, partial success in 4 and 1 target VT remained inducible. Programmed stimulation following ablation was not performed in 6 patients to avoid further haemodynamic stress. Potentially device-related adverse events occurred in 4 patients. In an unwell, high-risk patient group $68 \%$ of patients were free from VT or had a significantly reduced arrhythmia burden at 6 months follow up. 


\section{Bipolar and simultaneous ablation}

Bipolar ablation has grown in interest recently for the treatment of arrhythmias in difficult to reach myocardial locations by standard sequential unipolar ablation techniques. Bipolar ablation utilises 2 catheters on opposite sides of the myocardium, endocardial to epicardial or RV to LV in the IVS. One catheter will act as the return electrode for the RF energy from the other ablation catheter focussing RF energy between the two electrodes. ${ }^{97)}$

Recently Della Bella et al. ${ }^{105)}$ reported the safety and efficacy of bipolar ablation in a prospective pilot study of 21 patients with NICM and drug-refractory VT from the IVS. The bipolar ablation group was compared with patients with $<5 \mathrm{~mm}$ IVS wall thickness, or nonseptal VTs, or extra IVS substrate. Utilizing specialist software on the EAM system (Biosense Webster, Inc.) both catheter positions could be tracked, simultaneous real-time CF from both catheters, and distance measurements between catheter tip electrodes. Irrigated ablation catheters (Thermocool or Smarttouch; Biosense Webster, Inc.) were used at powers of 25-40 $\mathrm{W}$ up to 90 seconds in duration aiming for an impedance drop of 20-40 ohms. Of the 21 patients 14 presented with ES ( $n=14)$, isolated paroxysmal episodes $(n=4)$ and incessant VT $(\mathrm{n}=3)$. High density EAM confirmed the presence of low septal scar in all cases. Ablation was predominantly performed (19 out of 21) in the mid to basal IVS. Bipolar ablation terminated VT in 6 cases out of 17 with inducible VT. Tamponade occurred in one bipolar ablation patient with drainage and uneventful clinical course. No steam pops occurred in any procedures. Non-inducibility of the clinical VT was achieved in 20 of 21 patients (95\%) of cases. Noninducibility of any VT was achieved in 15 patients (71\%). At 25 months follow up VT recurred in 7 patients (33\%) with 2 VTs consistent with the clinical VT. While unable to prove superiority over traditional ablation approaches, the authors concluded that bipolar ablation allowed effective treatment of VT where standard methods had failed.

Igarashi et al., ${ }^{106)}$ reported acute and long-term results of bipolar ablation for VAs were reported in a retrospective multi-centre study. Eighteen patients across 7 centres had bipolar ablation after failing standard ablation approaches. Predominant sites for bipolar RF were targeted in the IVS (50\%), LV free wall (16\%) and LV summit (28\%). Three patients (17\%) had ICM and 15 patients ( $83 \%$ ) NICM. Irrigated ablation catheters were used with a custommade cable for the return catheter. Bipolar ablation was performed from 20-45W. Steam pops occurred during bipolar ablation of the IVS in 1 patient without cardiac tamponade or septal perforation. A separate patient had a left anterior descending artery occlusion on angiography following bipolar ablation. In 16 patients (89\%) and/or non-inducibility of all VAs was achieved with bipolar ablation. Eight patients (44\%) had VT recurrence at 12 months, and 2 further patients had late recurrence at longer follow up (range, 3-70 months). Burden of repetitive VT episodes ( $61 \%$ vs. $22 \%$ ) and VT (39\% vs. 5\%) was decreased following bipolar ablation but long-term VT recurrence was high (56\%).

Futyma et al., ${ }^{107)}$ have recently reported on bipolar ablation using an anatomical approach from the left pulmonary cusp (LPC) to the LVOT for LV summit arrhythmias. Three centres retrospectively reviewed 7 patients (5 PVCs and 2 VTs) that had failed standard RF techniques or had a reduction in VT burden. Mapping was attempted in the GCV and AIV in all patients. Activation times in the LPC were $16 \pm 11 \mathrm{~ms}$ similar to that of adjacent $\mathrm{LV}$ summit sites. One ablation catheter was placed in the LPC and the second catheter was positioned at the LVOT, aortic cusps or a subvalvular region below the level of the LPC located catheter. Care was taken to avoid the $\mathrm{LAD} \geq 5 \mathrm{~mm}$ with the LPC placed ablation catheter at all times. Bipolar ablation was performed at $30-40 \mathrm{~W}$ for up to 120 seconds. Four patients had acute 
termination of the PVC/VT with bipolar ablation. No steam pops or complications related to bipolar ablation were reported. During follow up (mean, 14 \pm 6 months; range, 8-21 months) 5 patients were asymptomatic without VT. VA burden was decreased from $31 \pm 13 \%$ PVCs per day before bipolar ablation to $4 \pm 5 \%$ PVC post ( $\mathrm{p}=0.0027$ ). An anatomical approach to bipolar ablation in normally inaccessible regions is a viable alternative in select patients.

In contrast to bipolar ablation, simultaneous unipolar ablation is applied by separate RF generators and ablation catheters operate independently of each other but have a bridged heating effect. ${ }^{108}$ An advantage of this approach is the ready availability of this technology in most electrophysiology laboratories without requiring specialist equipment or expertise in novel ablative therapies. Simultaneous unipolar RF also allows the independent titration of ablation power per catheter. ${ }^{109)}$

Simultaneous ablation has previously been reported clinically for intramural foci from the LVOT. ${ }^{110)}$ Recently, outcomes of simultaneous ablation have been reported for intramural septal ablation in NICM patients. ${ }^{109)} \mathrm{A}$ total of 6 patients underwent simultaneous ablation either during an initial $\mathrm{RF}$ ablation procedure $(\mathrm{n}=2)$ or at a repeat procedure $(\mathrm{n}=4)$. All six patients had mid myocardial septal late gadolinium enhancement on cMR and low unipolar voltage and abnormal electrograms on EAM. In three patient's sequential biventricular RF was delivered during VT with late termination followed by VT re-inducible with programmed extra stimulus. VT was terminated in four cases when simultaneous RF was applied across the septum and in two cases previously inducible VT was rendered non-inducible following substrate ablation at best pace map site and its adjacent IVS location. Mean distances between the ablation catheters during simultaneous ablation was $13.3 \pm 4 \mathrm{~mm}$ (range, 7.8-20.1 mm). No acute complications were reported during the procedures or steam pops during either sequential or simultaneous ablations. At a median follow-up of 20 months (range, 13-20 months) 2 patients (33\%) had VT recurrence with one patient having late recurrence at 20 months.

\section{Stereotactic body radiation therapy}

SBRT has been used for the treatment of benign and malignant delivers precise radiation to target regions in the body with minimal damage to adjacent tissue. In 2017, Cuculich et al. ${ }^{111}$ published pilot data of a completely non-invasive approach to the treatment of VT utilising the previously mentioned ECGi vest, CT or CMR and SBRT of 25 Gy. Initially 5 patients with refractory VT (at least 3 episodes in prior 3 months) after at least two AADs and one failed CA underwent SBRT. After an initial 6-week blanking period, patients had a 99.9\% reduction in VT episodes and no effect on left ventricular systolic function.

A systematic review of radio-ablation therapy for arrhythmias summarising electrophysiological and histopathological effects has been reported. ${ }^{122}$ Ten clinical studies with a total of 41 patients were included with $90 \%$ treated for VT. Photon radiotherapy was used in all patients and doses ranged from $24 \mathrm{~Gy}$ in 3 fractions to single fraction 24-25 Gy. All publications showed a reduction in VT episodes within the first month of treatment, with 4 publications reporting pre- and post-therapy VA burden, after a blanking period, of $>85 \%$. In 3 publications with a total of 16 patients late recurrence ( $>6$ months after therapy) was reported in 7 patients $(46 \%)$ with a follow up $>6$ months duration. ${ }^{111113) 114)}$ Only minor side effects such as fatigue, hypotension, and nausea post SBRT were reported immediately after treatment. ${ }^{1131115)}$ During follow up, 7 patients died, and 2 of them were arrhythmia related. Two more patients died outside the follow up period in the Encore-VT trial with worsening heart failure and VT recurrence. ${ }^{112}$ 


\section{SYMPATHECTOMY}

Cardiac sympathetic denervation (CSD) is an established treatment for VAs in patients with some forms of congenital LQTS and catecholaminergic polymorphic ventricular tachycardia (CPVT) ${ }^{110) 117)}$ A meta-analysis of the role of CSD in the management of VAs has been reported. ${ }^{118)}$ Fourteen non-randomized studies with 311 patients and a mean follow up of $15 \pm 10.7$ months were included. The mean age of the patients was $49 \pm 12$ years. The predominant etiology was NICM. Analysis of all causes of arrhythmias showed the pooled rate of freedom from VT after CSD was $60 \%$. If LQTS and CPVT patients were excluded, the freedom from VT rate was reduced to $50 \%$. After CSD, mean total of ICD shocks was reduced for all causes of arrhythmia by 3.01 ( $95 \% \mathrm{CI}, 1.09-4.94 ; \mathrm{p}=0.002)$ and 0.97 ( $95 \% \mathrm{CI}$, 0.41-1.5; $\mathrm{p}=0.001$ ) when LQTS and CPVT were excluded. Adverse events associated with the procedure were pneumothorax (3.2\%) and Horner-Bernard syndrome $(2.2 \%)$. CSD may be a viable option in patients with VT refractory to AADs and CA, though the presence of heart failure class III and IV, slow VT $>400 \mathrm{~ms}$, extensive scarring, left sided only CSD and lack of a sympathetic trigger of their arrhythmias has reduced or negligible benefit. ${ }^{18-120)}$

A less invasive approach has been achieved by high thoracic epidural anaesthesia at the T1-T2 or T2-T3 level causing stellate ganglion blockade (SGB) as an acute bridge to longerterm therapy. A systematic review has been reported on the use of SGB and its role in ES management. ${ }^{121)}$ Twenty-three studies between 1976 and 2016 identified 38 patients. Only $15.8 \%$ of patients were treated with CA prior to SGB. VA burden $(12.4 \pm 8.8$ vs. $1.04 \pm 2.12$ episodes/day, $\mathrm{p} \leq 0.001)$ and external or ICD shocks $(10.0 \pm 9.1$ vs. $0.05 \pm 0.22$ episodes/day, $\mathrm{p} \leq 0.01$ ) were reduced significantly in patients with ES after SGB was administered. Complete VA suppression occurred in $63 \%$ of patients. Depending on the anaesthetic used the SGB effect lasted from 6 hours to 4 weeks. SGB administration benefitted patients regardless of LV function status (normal function, mild, moderate and severe LV dysfunction) and aetiology of cardiomyopathy. A reduction in VA burden by $<50 \%$ only occurred in $10 \%$.

Renal denervation (RDN) for VAs is a burgeoning area complimentary to direct myocardial ablation. RDN is an alternative method to target the sympathetic nervous system by interruption of abnormal afferent signalling that is associated with increased renal nerve activity and noradrenaline release. ${ }^{122}$ A systematic review and meta-analysis on RDN for the management of drug-refractory VA is available in press by Hawson et al. ${ }^{122}$ In the review 7 studies were identified with a total of 121 pooled patients. Patients were predominantly male $(76 \%)$, on a beta-blocker $(99 \%)$ and/or amiodarone $(79 \%)$, and $46 \%$ had previously undergone a CA. All patients had SHD, 55\% ICM and 45\% NICM. The analysis demonstrated a significant effect of RDN in reducing ICD therapies with a standardised mean difference (SMD) of $-3.11(\mathrm{p}<0.001)$. RDN also reduced the number of VA episodes (SMD -2.13, $\mathrm{p}<0.001$ ), anti-tachycardia pacing episodes ( $\mathrm{SMD}-2.82, \mathrm{p}=0.002$ ) and shocks ( $\mathrm{SMD}-2.82, \mathrm{p}=0.002$ ). Across publications, $\mathrm{RDN}$ appeared safe with a low risk of complications acutely or late. Whilst randomised data is lacking, RDN appears as an effective treatment for refractory VAs and electrical storms.

\section{ADAPTATION OF EXISTING TECHNIQUES TO IMPROVE VENTRICULAR ARRHYTHMIAS ABLATION OUTCOMES}

There have been recent advancements to refine existing techniques for induction, mapping, and ablation in an attempt to improve the efficiency and efficacy of VT ablation procedures. 

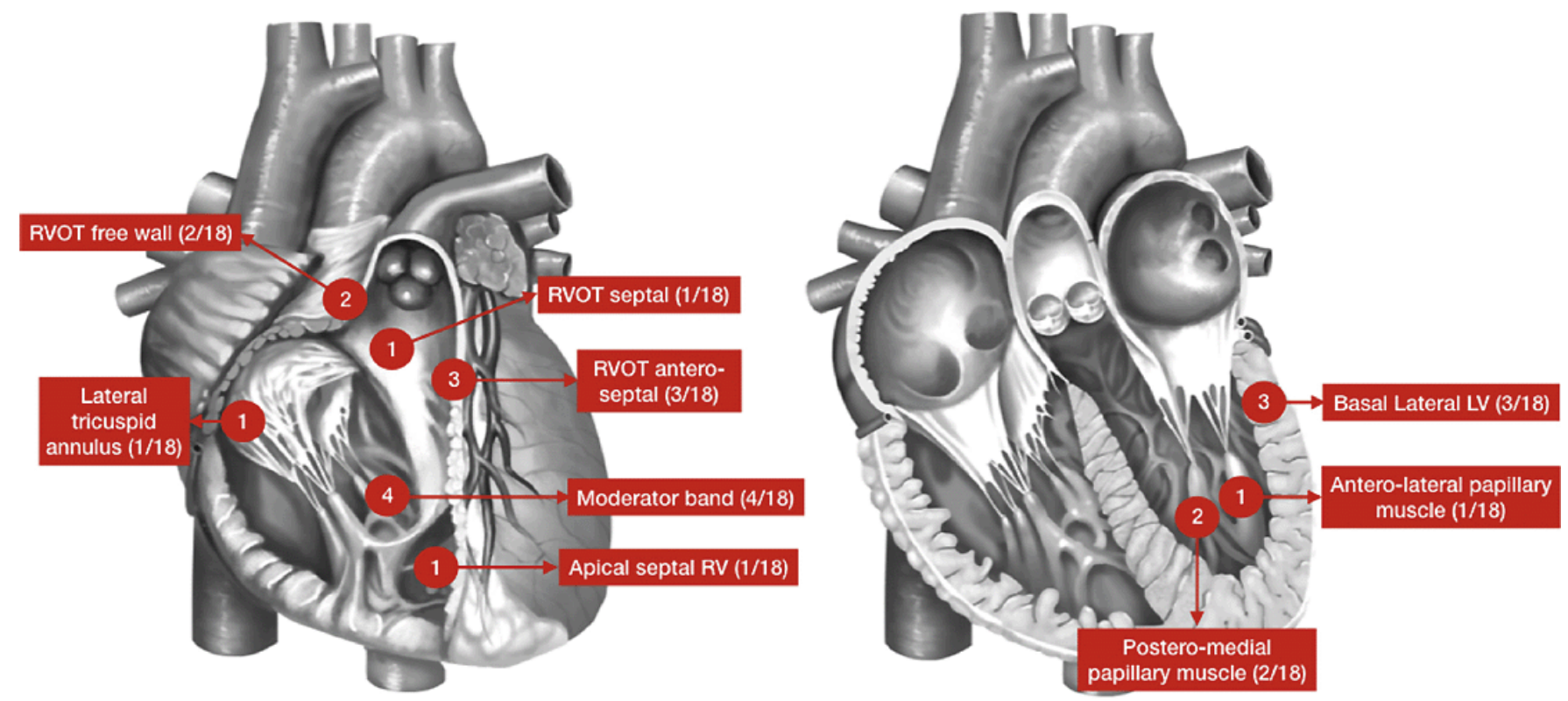

Figure 4. Focal ventricular tachycardia in patients with structural heart disease.

Reprinted from Robert D. Anderson, Geoffrey Lee, Ivana Trivic, Timothy Campbell, Timmy Pham, Chrishan Nalliah, Eddy Kizana, Stuart P. Thomas, Siddharth J. Trivedi, Troy Watts, Jonathan Kalman and Saurabh Kumar, Focal Ventricular Tachycardias in Structural Heart Disease: Prevalence, Characteristics, and Clinical Outcomes After Catheter Ablation. JACC Clinical Electrophysiology 2020;6(1):56-69, with permission from Elsevier.

$\mathrm{LV}=$ left ventricle; RV = right ventricle; RVOT = right ventricular outflow tract.

Anderson et al., ${ }^{1}$ challenged the concept VT in patients with SHD is always scar related. Focal VT, remote from SHD substrate was induced in $16 \%$ of patients with burst pacing and/or high dose isoproterenol infusion (up to $40 \mu \mathrm{g} / \mathrm{min}$ ). Focal VTs showed repetitive failure to terminate with anti-tachycardia pacing $(69 \%)$ or ICD shock (56\%) and $88 \%$ of the focal VT matched the clinical VT. Sites of focal VT were from areas traditionally consistent with sites of 'idiopathic' VT such as RVOT (33\%), moderator band (22\%) and papillary muscles (17\%) apical septum $(6 \%)$ and tricuspid annulus (6\%) in the RV and mitral annulus $(16 \%)$ in the LV. In the remaining $10 \%$, sites of VT were at the border zone of scar (Figure 4). The fact that these VTs required isoprenaline and burst pacing for induction, suggest a focal mechanism. Furthermore, these VTs would have been missed if high dose isoprenaline testing and burst pacing were not used. These VTs may indeed explain the modest recurrence rates of ablation of VT for SHD.

Induction and identification of these VTs are paramount for success. To this end Campbell et al., ${ }^{123}$ investigated induction protocols for SHD VT that included the current guidelines directed 3 extra-stimulus approach versus an extensive protocol of 4 extra-stimulus and burst $\mathrm{RV}$ pacing. In 62 patients an extensive testing protocol induced $11-17 \%$ more VT than the limited protocol before and after ablation without inducing a significant difference in VF or fast VT compared to the limited protocol. Importantly, patients that were non-inducible post ablation with an extensive protocol had better VA-free survival at 12 months ( $82 \%$ vs. $43 \%$, $\mathrm{p}=0.03$ ) and better survival free of VA, transplantation and mortality at 12 months ( $82 \%$ vs. $46 \%, p=0.02$ ). These findings suggest a prognostic benefit of testing with the more extensive protocol after CA of VT.

Pacing along an ablation line to confirm loss of capture for efficacy is a concept introduced early in the electrophysiology literature. Soejima et al. ${ }^{124)}$ showed pacing within low-voltage infarct regions could locate reentry circuit isthmuses by defining their borders and pacing 
substrate post ablation to confirm it is unexcitable in SHD. Steven et al. ${ }^{125}$ showed pacing to confirm loss of electrical excitability along an ablation line was highly efficacious in atrial fibrillation. Whilst simultaneous pacing and ablating has been reported as safe and without induction of VF in atrial fibrillation ablations its use in VAs has not been investigated. ${ }^{126)}$ Anderson et al., ${ }^{19)}$ recently combined pacing for efficacy confirmation and simultaneous ablation during substrate ablation of VT in 10 patients. ICM was present in $90 \%$ of patients and $70 \%$ with VT storm. All patients underwent scar homogenisation with electrical inexcitability to pacing (10 mA, 9 ms pulse width). Standard ablation parameters (CF and impedance reduction) were not impaired during simultaneous pacing and ablation in over $>1,100$ ablations. Using an extensive induction protocol $97 \%$ of VTs were no longer inducible with this method. All patients remained VA-free during follow up (median, 182 days; IQR, $65-347)$ though 1 patient died without recurrence of VA. This preliminary data prove safety and efficacy at midterm follow up and requires larger studies and different SHD aetiologies for widespread translation to clinical practice.

\section{FUTURE DIRECTIONS}

The lack of a randomised trial of patients with NICM and recurrent VT treated with CA is a large void in the field. Likewise, CF sensing catheters have not been extensively studied with relation to outcomes in randomised trials. A question remains as to whether the new techniques for targeted substrate ablation by pre-procedural imaging guided by dechanneling, ILAM, Ripple mapping or DEEP will provide a better outcome than a scar homogenisation approach is unknown. Substrate mapping techniques will be further refined when MEM catheter electrode contact algorithms are widespread across all EAM systems. The burgeoning field of cardiac arrhythmia radio-ablation therapy needs further work on pathophysiology and histological effects as well as the integration of current invasive mapping system data into an exportable format for radiation therapy planning tools.

\section{REFERENCES}

1. Anderson RD, Lee G, Trivic I, et al. Focal ventricular tachycardias in structural heart disease: prevalence, characteristics, and clinical outcomes after catheter ablation. JACC Clin Electrophysiol 2020;6:56-69. PUBMED | CROSSREF

2. Nakahara S, Tung R, Ramirez RJ, et al. Characterization of the arrhythmogenic substrate in ischemic and nonischemic cardiomyopathy implications for catheter ablation of hemodynamically unstable ventricular tachycardia. J Am Coll Cardiol 2010;55:2355-65. PUBMED | CROSSREF

3. Shirai Y, Liang JJ, Santangeli P, et al. Comparison of the ventricular tachycardia circuit between patients with ischemic and nonischemic cardiomyopathies: detailed characterization by entrainment. Circ Arrhythm Electrophysiol 2019;12:e007249. PUBMED | CROSSREF

4. Poole JE, Johnson GW, Hellkamp AS, et al. Prognostic importance of defibrillator shocks in patients with heart failure. N Engl J Med 2008;359:1009-17. PUBMED | CROSSREF

5. Schron EB, Exner DV, Yao Q et al. Quality of life in the antiarrhythmics versus implantable defibrillators trial: impact of therapy and influence of adverse symptoms and defibrillator shocks. Circulation 2002;105:589-94. PUBMED | CROSSREF

6. Strickberger SA, Canby R, Cooper J, et al. Association of antitachycardia pacing or shocks with survival in 69,000 patients with an implantable defibrillator. J Cardiovasc Electrophysiol 2017;28:416-22.

PUBMED | CROSSREF 
7. Al-Khatib SM, Stevenson WG, Ackerman MJ, et al. 2017 AHA/ACC/HRS guideline for management of patients with ventricular arrhythmias and the prevention of sudden cardiac death: executive summary: a report of the American College of Cardiology/American Heart Association Task Force on Clinical Practice Guidelines and the Heart Rhythm Society. Heart Rhythm 2018;15:e190-252. PUBMED | CROSSREF

8. Bokhari F, Newman D, Greene M, Korley V, Mangat I, Dorian P. Long-term comparison of the implantable cardioverter defibrillator versus amiodarone: eleven-year follow-up of a subset of patients in the Canadian Implantable Defibrillator Study (CIDS). Circulation 2004;110:112-6. PUBMED | CROSSREF

9. Romero J, Stevenson WG, Fujii A, et al. Impact of number of oral antiarrhythmic drug failures before referral on outcomes following catheter ablation of ventricular tachycardia. JACC Clin Electrophysiol 2018;4:810-9. PUBMED | CROSSREF

10. Dinov B, Fiedler L, Schönbauer R, et al. Outcomes in catheter ablation of ventricular tachycardia in dilated nonischemic cardiomyopathy compared with ischemic cardiomyopathy: results from the Prospective Heart Centre of Leipzig VT (HELP-VT) Study. Circulation 2014;129:728-36. PUBMED | CROSSREF

11. Anderson RD, Lee G, Prabhu M, et al. Ten-year trends in catheter ablation for ventricular tachycardia vs other interventional procedures in Australia. J Cardiovasc Electrophysiol 2019;30:2353-61. PUBMED | CROSSREF

12. Sapp JL, Wells GA, Parkash R, et al. Ventricular tachycardia ablation versus escalation of antiarrhythmic drugs. N Engl J Med 2016;375:111-21. PUBMED | CROSSREF

13. Parkash R, Nault I, Rivard L, et al. Effect of baseline antiarrhythmic drug on outcomes with ablation in ischemic ventricular tachycardia: a VANISH substudy (Ventricular Tachycardia Ablation Versus Escalated Antiarrhythmic Drug Therapy in Ischemic Heart Disease). Circ Arrhythm Electrophysiol 2018;11:e005663. PUBMED | CROSSREF

14. Anderson RD, Ariyarathna N, Lee G, et al. Catheter ablation versus medical therapy for treatment of ventricular tachycardia associated with structural heart disease: systematic review and meta-analysis of randomized controlled trials and comparison with observational studies. Heart Rhythm 2019;16:1484-91. PUBMED | CROSSREF

15. Muser D, Santangeli P, Castro SA, et al. Long-term outcome after catheter ablation of ventricular tachycardia in patients with nonischemic dilated cardiomyopathy. Circ Arrhythm Electrophysiol 2016;9:9. PUBMED | CROSSREF

16. Kumar S, Romero J, Mehta NK, et al. Long-term outcomes after catheter ablation of ventricular tachycardia in patients with and without structural heart disease. Heart Rhythm 2016;13:1957-63. PUBMED | CROSSREF

17. Maron BJ, Towbin JA, Thiene G, et al. Contemporary definitions and classification of the cardiomyopathies: an American Heart Association Scientific Statement from the Council on Clinical Cardiology, Heart Failure and Transplantation Committee; Quality of Care and Outcomes Research and Functional Genomics and Translational Biology Interdisciplinary Working Groups; and Council on Epidemiology and Prevention. Circulation 2006;113:1807-16. PUBMED | CROSSREF

18. Di Biase L, Santangeli P, Burkhardt DJ, et al. Endo-epicardial homogenization of the scar versus limited substrate ablation for the treatment of electrical storms in patients with ischemic cardiomyopathy. J Am Coll Cardiol 2012;60:132-41. PUBMED | CROSSREF

19. Anderson RD, Lee G, Campbell T, et al. Scar nonexcitability using simultaneous pacing for substrate ablation of ventricular tachycardia. Pacing Clin Electrophysiol. 2020 [Epub ahead of print]. PUBMED | CROSSREF

20. Gökoğlan Y, Mohanty S, Gianni C, et al. Scar homogenization versus limited-substrate ablation in patients with nonischemic cardiomyopathy and ventricular tachycardia. J Am Coll Cardiol 2016;68:1990-8. PUBMED | CROSSREF

21. Bennett RG, Haqqani HM, Berruezo A, et al. Arrhythmogenic cardiomyopathy in 2018-2019: ARVC/ALVC or both? Heart Lung Circ 2019;28:164-77. PUBMED | CROSSREF

22. Mahida S, Venlet J, Saguner AM, et al. Ablation compared with drug therapy for recurrent ventricular tachycardia in arrhythmogenic right ventricular cardiomyopathy: results from a multicenter study. Heart Rhythm 2019;16:536-43.

PUBMED | CROSSREF 
23. Garcia FC, Bazan V, Zado ES, Ren JF, Marchlinski FE. Epicardial substrate and outcome with epicardial ablation of ventricular tachycardia in arrhythmogenic right ventricular cardiomyopathy/dysplasia. Circulation 2009;120:366-75. PUBMED | CROSSREF

24. Romero J, Patel K, Briceno D, et al. Endo-epicardial ablation vs endocardial ablation for the management of ventricular tachycardia in arrhythmogenic right ventricular cardiomyopathy: a systematic review and meta-analysis. J Cardiovasc Electrophysiol 2020;31:2022-31. PUBMED | CROSSREF

25. Kumar S, Barbhaiya C, Nagashima K, et al. Ventricular tachycardia in cardiac sarcoidosis: characterization of ventricular substrate and outcomes of catheter ablation. Circ Arrhythm Electrophysiol 2015;8:87-93. PUBMED | CROSSREF

26. Papageorgiou N, Providência R, Bronis K, et al. Catheter ablation for ventricular tachycardia in patients with cardiac sarcoidosis: a systematic review. Europace 2018;20:682-91. PUBMED | CROSSREF

27. Nademanee K, Veerakul G, Chandanamattha P, et al. Prevention of ventricular fibrillation episodes in Brugada syndrome by catheter ablation over the anterior right ventricular outflow tract epicardium. Circulation 2011;123:1270-9. PUBMED | CROSSREF

28. Nademanee K, Haissaguerre M, Hocini M, et al. Mapping and ablation of ventricular fibrillation associated with early repolarization syndrome. Circulation 2019;140:1477-90. PUBMED | CROSSREF

29. Pappone C, Brugada J, Vicedomini G, et al. Electrical substrate elimination in 135 consecutive patients with Brugada syndrome. Circ Arrhythm Electrophysiol 2017;10:e005053. PUBMED | CROSSREF

30. Haïssaguerre M, Shoda M, Jaïs P, et al. Mapping and ablation of idiopathic ventricular fibrillation. Circulation 2002;106:962-7. PUBMED | CROSSREF

31. Knecht S, Sacher F, Wright M, et al. Long-term follow-up of idiopathic ventricular fibrillation ablation: a multicenter study. J Am Coll Cardiol 2009;54:522-8. PUBMED | CROSSREF

32. Nogami A. Mapping and ablating ventricular premature contractions that trigger ventricular fibrillation: trigger elimination and substrate modification. J Cardiovasc Electrophysiol 2015;26:110-5. PUBMED | CROSSREF

33. Haïssaguerre M, Extramiana F, Hocini M, et al. Mapping and ablation of ventricular fibrillation associated with long-QT and Brugada syndromes. Circulation 2003;108:925-8. PUBMED | CROSSREF

34. Haïssaguerre M, Duchateau J, Dubois R, et al. Idiopathic ventricular fibrillation: role of Purkinje system and microstructural myocardial abnormalities. JACC Clin Electrophysiol 2020;6:591-608. PUBMED | CROSSREF

35. Nash MP, Mourad A, Clayton RH, et al. Evidence for multiple mechanisms in human ventricular fibrillation. Circulation 2006;114:536-42. PUBMED | CROSSREF

36. Nair K, Umapathy K, Farid T, et al. Intramural activation during early human ventricular fibrillation. Circ Arrhythm Electrophysiol 2011;4:692-703. PUBMED | CROSSREF

37. Nakamura T, Schaeffer B, Tanigawa S, et al. Catheter ablation of polymorphic ventricular tachycardia/ fibrillation in patients with and without structural heart disease. Heart Rhythm 2019;16:1021-7. PUBMED | CROSSREF

38. Santangeli P, Frankel DS, Tung R, et al. Early mortality after catheter ablation of ventricular tachycardia in patients with structural heart disease. J Am Coll Cardiol 2017;69:2105-15. PUBMED | CROSSREF

39. Vergara P, Tzou WS, Tung R, et al. Predictive score for identifying survival and recurrence risk profiles in patients undergoing ventricular tachycardia ablation: the I-VT score. Circ Arrhythm Electrophysiol 2018;11:e006730. PUBMED | CROSSREF

40. Muser D, Castro SA, Liang JJ, Santangeli P. Identifying risk and management of acute haemodynamic decompensation during catheter ablation of ventricular tachycardia. Arrhythm Electrophysiol Rev 2018;7:282-7. PUBMED | CROSSREF

41. Dickfeld T, Calkins H, Zviman M, et al. Anatomic stereotactic catheter ablation on three-dimensional magnetic resonance images in real time. Circulation 2003;108:2407-13. PUBMED | CROSSREF 
42. Solomon SB, Dickfeld T, Calkins H. Real-time cardiac catheter navigation on three-dimensional CT images. J Interv Card Electrophysiol 2003;8:27-36.

PUBMED | CROSSREF

43. Berruezo A, Fernández-Armenta J, Andreu D, et al. Scar dechanneling: new method for scar-related left ventricular tachycardia substrate ablation. Circ Arrhythm Electrophysiol 2015;8:326-36. PUBMED | CROSSREF

44. Berruezo A, Fernández-Armenta J, Mont L, et al. Combined endocardial and epicardial catheter ablation in arrhythmogenic right ventricular dysplasia incorporating scar dechanneling technique. Circ Arrhythm Electrophysiol 2012;5:111-21. PUBMED | CROSSREF

45. Ustunkaya T, Desjardins B, Liu B, et al. Association of regional myocardial conduction velocity with the distribution of hypoattenuation on contrast-enhanced perfusion computed tomography in patients with postinfarct ventricular tachycardia. Heart Rhythm 2019;16:588-94. PUBMED | CROSSREF

46. Ustunkaya T, Desjardins B, Wedan R, et al. Epicardial conduction speed, electrogram abnormality, and computed tomography attenuation associations in arrhythmogenic right ventricular cardiomyopathy. JACC Clin Electrophysiol 2019;5:1158-67. PUBMED | CROSSREF

47. Mahida S, Sacher F, Dubois R, et al. Cardiac imaging in patients with ventricular tachycardia. Circulation 2017;136:2491-507. PUBMED | CROSSREF

48. Piers SR, Tao Q, van Huls van Taxis CF, Schalij MJ, van der Geest RJ, Zeppenfeld K. Contrast-enhanced MRI-derived scar patterns and associated ventricular tachycardias in nonischemic cardiomyopathy: implications for the ablation strategy. Circ Arrhythm Electrophysiol 2013;6:875-83. PUBMED | CROSSREF

49. Yamashita S, Sacher F, Hooks DA, et al. Myocardial wall thinning predicts transmural substrate in patients with scar-related ventricular tachycardia. Heart Rhythm 2017;14:155-63. PUBMED | CROSSREF

50. Yamashita S, Sacher F, Mahida S, et al. Image integration to guide catheter ablation in scar-related ventricular tachycardia. J Cardiovasc Electrophysiol 2016;27:699-708. PUBMED | CROSSREF

51. Andreu D, Ortiz-Pérez JT, Fernández-Armenta J, et al. 3D delayed-enhanced magnetic resonance sequences improve conducting channel delineation prior to ventricular tachycardia ablation. Europace 2015;17:938-45. PUBMED | CROSSREF

52. Andreu D, Penela D, Acosta J, et al. Cardiac magnetic resonance-aided scar dechanneling: influence on acute and long-term outcomes. Heart Rhythm 2017;14:1121-8. PUBMED | CROSSREF

53. Roca-Luque I, Van Breukelen A, Alarcon F, et al. Ventricular scar channel entrances identified by new wideband cardiac magnetic resonance sequence to guide ventricular tachycardia ablation in patients with cardiac defibrillators. Europace 2020;22:598-606.

PUBMED | CROSSREF

54. Penela D, Martínez M, Fernández-Armenta J, et al. Influence of myocardial scar on the response to frequent premature ventricular complex ablation. Heart 2019;105:378-83. PUBMED

55. Cury RC, Nieman K, Shapiro MD, et al. Comprehensive assessment of myocardial perfusion defects, regional wall motion, and left ventricular function by using 64-section multidetector CT. Radiology 2008;248:466-75. PUBMED | CROSSREF

56. Esposito A, Palmisano A, Antunes S, et al. Cardiac CT with delayed enhancement in the characterization of ventricular tachycardia structural substrate: relationship between CT-segmented scar and electroanatomic mapping. JACC Cardiovasc Imaging 2016;9:822-32. PUBMED | CROSSREF

57. Deno DC, Balachandran R, Morgan D, Ahmad F, Masse S, Nanthakumar K. Orientation-independent catheter-based characterization of myocardial activation. IEEE Trans Biomed Eng 2017;64:1067-77. PUBMED | CROSSREF

58. Massé S, Magtibay K, Jackson N, et al. Resolving myocardial activation with novel omnipolar electrograms. Circ Arrhythm Electrophysiol 2016;9:e004107. PUBMED | CROSSREF 
59. Magtibay K, Massé S, Asta J, et al. Physiological assessment of ventricular myocardial voltage using omnipolar electrograms. J Am Heart Assoc 2017;6:e006447. PUBMED | CROSSREF

60. Haldar SK, Magtibay K, Porta-Sanchez A, et al. Resolving bipolar electrogram voltages during atrial fibrillation using omnipolar mapping. Circ Arrhythm Electrophysiol 2017;10:e005018. PUBMED | CROSSREF

61. Porta-Sánchez A, Magtibay K, Nayyar S, et al. Omnipolarity applied to equi-spaced electrode array for ventricular tachycardia substrate mapping. Europace 2019;21:813-21. PUBMED | CROSSREF

62. Irie T, Yu R, Bradfield JS, et al. Relationship between sinus rhythm late activation zones and critical sites for scar-related ventricular tachycardia: systematic analysis of isochronal late activation mapping. Circ Arrhythm Electrophysiol 2015;8:390-9. PUBMED | CROSSREF

63. Aziz Z, Shatz D, Raiman M, et al. Targeted ablation of ventricular tachycardia guided by wavefront discontinuities during sinus rhythm: a new functional substrate mapping strategy. Circulation 2019;140:1383-97. PUBMED | CROSSREF

64. Linton NW, Koa-Wing M, Francis DP, et al. Cardiac ripple mapping: a novel three-dimensional visualization method for use with electroanatomic mapping of cardiac arrhythmias. Heart Rhythm 2009;6:1754-62. PUBMED | CROSSREF

65. Jamil-Copley S, Vergara P, Carbucicchio C, et al. Application of ripple mapping to visualize slow conduction channels within the infarct-related left ventricular scar. Circ Arrhythm Electrophysiol 2015;8:76-86. PUBMED | CROSSREF

66. Luther V, Linton NW, Jamil-Copley S, et al. A Prospective study of ripple mapping the post-infarct ventricular scar to guide substrate ablation for ventricular tachycardia. Circ Arrhythm Electrophysiol 2016;9:e004072. PUBMED | CROSSREF

67. Jackson N, Gizurarson S, Viswanathan K, et al. Decrement evoked potential mapping: basis of a mechanistic strategy for ventricular tachycardia ablation. Circ Arrhythm Electrophysiol 2015;8:1433-42. PUBMED | CROSSREF

68. Porta-Sánchez A, Jackson N, Lukac P, et al. Multicenter study of ischemic ventricular tachycardia ablation with decrement-evoked potential (DEEP) mapping with extra stimulus. JACC Clin Electrophysiol 2018;4:307-15. PUBMED | CROSSREF

69. Rudy Y, Lindsay BD. Electrocardiographic imaging of heart rhythm disorders: from bench to bedside. Card Electrophysiol Clin 2015;7:17-35.

PUBMED | CROSSREF

70. Cuculich PS, Zhang J, Wang Y, et al. The electrophysiological cardiac ventricular substrate in patients after myocardial infarction: noninvasive characterization with electrocardiographic imaging. J Am Coll Cardiol 2011;58:1893-902. PUBMED | CROSSREF

71. Zhang J, Sacher F, Hoffmayer K, et al. Cardiac electrophysiological substrate underlying the ECG phenotype and electrogram abnormalities in Brugada syndrome patients. Circulation 2015;131:1950-9. PUBMED | CROSSREF

72. Rudic B, Chaykovskaya M, Tsyganov A, et al. Simultaneous non-invasive epicardial and endocardial mapping in patients with Brugada syndrome: new insights into arrhythmia mechanisms. J Am Heart Assoc 2016;5:e004095. PUBMED | CROSSREF

73. Leong KM, Ng FS, Roney C, et al. Repolarization abnormalities unmasked with exercise in sudden cardiac death survivors with structurally normal hearts. J Cardiovasc Electrophysiol 2018;29:115-26. PUBMED | CROSSREF

74. Graham AJ, Orini M, Zacur E, et al. Evaluation of ECG imaging to map hemodynamically stable and unstable ventricular arrhythmias. Circ Arrhythm Electrophysiol 2020;13:e007377. PUBMED | CROSSREF

75. Sapp JL, Zhou S, Wang L. Mapping ventricular tachycardia with electrocardiographic imaging. Circ Arrhythm Electrophysiol 2020;13:e008255. PUBMED | CROSSREF

76. Cronin EM, Bogun FM, Maury P, et al. 2019 HRS/EHRA/APHRS/LAHRS expert consensus statement on catheter ablation of ventricular arrhythmias. Europace 2019;21:1143-4.

PUBMED | CROSSREF 
77. Campbell T, Trivic I, Bennett RG, et al. Catheter ablation of ventricular arrhythmia guided by a highdensity grid catheter. J Cardiovasc Electrophysiol 2020;31:474-84.

PUBMED | CROSSREF

78. Okubo K, Frontera A, Bisceglia C, et al. Grid Mapping Catheter for Ventricular Tachycardia Ablation. Circ Arrhythm Electrophysiol 2019;12:e007500.

PUBMED | CROSSREF

79. St. Jude Medical, Inc. Instructions for Use: Ensite Precision ${ }^{\mathrm{TM}}$ Cardiac Mapping System Model EE3000. Software Version 2.0. Saint Paul (MN): St. Jude Medical, Inc.; 2016.

80. Takigawa M, Relan J, Martin R, et al. Detailed analysis of the relation between bipolar electrode spacing and far- and near-field electrograms. JACC Clin Electrophysiol 2019;5:66-77. PUBMED | CROSSREF

81. Barkagan M, Sroubek J, Shapira-Daniels A, et al. A novel multielectrode catheter for high-density ventricular mapping: electrogram characterization and utility for scar mapping. Europace 2020;22:440-9. PUBMED | CROSSREF

82. Sroubek J, Rottmann M, Barkagan M, et al. A novel octaray multielectrode catheter for high-resolution atrial mapping: electrogram characterization and utility for mapping ablation gaps. J Cardiovasc Electrophysiol 2019;30:749-57. PUBMED | CROSSREF

83. Viswanathan K, Mantziari L, Butcher C, et al. Evaluation of a novel high-resolution mapping system for catheter ablation of ventricular arrhythmias. Heart Rhythm 2017;14:176-83.

PUBMED | CROSSREF

84. Liu M, Jiang J, Su C, et al. Electrophysiological characteristics of the earliest activation site in idiopathic right ventricular outflow tract arrhythmias under mini-electrode mapping. J Cardiovasc Electrophysiol 2019;30:642-50. PUBMED | CROSSREF

85. Price A, Leshen Z, Hansen J, et al. Novel ablation catheter technology that improves mapping resolution and monitoring of lesion maturation. J Innov Card Rhythm Manag 2012;3:599-609. CROSSREF

86. Münkler P, Gunawardene MA, Jungen C, et al. Local impedance guides catheter ablation in patients with ventricular tachycardia. J Cardiovasc Electrophysiol 2020;31:61-9. PUBMED | CROSSREF

87. Leshem E, Tschabrunn CM, Jang J, et al. High-resolution mapping of ventricular scar: evaluation of a novel integrated multielectrode mapping and ablation catheter. JACC Clin Electrophysiol 2017;3:220-31. PUBMED | CROSSREF

88. Glashan CA, Tofig BJ, Tao Q, et al. Multisize electrodes for substrate identification in ischemic cardiomyopathy: validation by integration of whole heart histology. JACC Clin Electrophysiol 2019;5:1130-40. PUBMED | CROSSREF

89. Tilz RR, Makimoto H, Lin T, et al. In vivo left-ventricular contact force analysis: comparison of antegrade transseptal with retrograde transaortic mapping strategies and correlation of impedance and electrical amplitude with contact force. Europace 2014;16:1387-95. PUBMED | CROSSREF

90. Elsokkari I, Sapp JL, Doucette S, et al. Role of contact force in ischemic scar-related ventricular tachycardia ablation; optimal force required and impact of left ventricular access route. J Interv Card Electrophysiol 2018;53:323-31.

PUBMED | CROSSREF

91. Jesel L, Sacher F, Komatsu Y, et al. Characterization of contact force during endocardial and epicardial ventricular mapping. Circ Arrhythm Electrophysiol 2014;7:1168-73. PUBMED | CROSSREF

92. Mizuno H, Vergara P, Maccabelli G, et al. Contact force monitoring for cardiac mapping in patients with ventricular tachycardia. J Cardiovasc Electrophysiol 2013;24:519-24. PUBMED | CROSSREF

93. Aras D, Özcan F, Çay S, Özeke Ö, Kara M, Topaloğlu S. Endo/epicardial ablation of ventricular arrhythmias with contact force-sensing catheters in arrhythmogenic right ventricular dysplasia/cardiomyopathy. Anatol J Cardiol 2019;21:187-95. PUBMED | CROSSREF

94. Hendriks AA, Akca F, Dabiri Abkenari L, et al. Safety and clinical outcome of catheter ablation of ventricular arrhythmias using contact force sensing: consecutive case series. J Cardiovasc Electrophysiol 2015;26:1224-9.

PUBMED | CROSSREF 
95. Elbatran AI, Li A, Gallagher MM, et al. Contact force sensing in ablation of ventricular arrhythmias using a 56-hole open-irrigation catheter: a propensity-matched analysis. J Interv Card Electrophysiol 2020 [Epub ahead of print]. PUBMED | CROSSREF

96. de Vries LJ, Hendriks AA, Yap SC, Theuns DAMJ, van Domburg RT, Szili-Torok T. Procedural and longterm outcome after catheter ablation of idiopathic outflow tract ventricular arrhythmias: comparing manual, contact force, and magnetic navigated ablation. Europace 2018;20:ii22-7. PUBMED | CROSSREF

97. Sandhu A, Nguyen DT. Forging ahead: update on radiofrequency ablation technology and techniques. $J$ Cardiovasc Electrophysiol 2020;31:360-9. PUBMED | CROSSREF

98. Nguyen DT, Gerstenfeld EP, Tzou WS, et al. Radiofrequency ablation using an open irrigated electrode cooled with half-normal saline. JACC Clin Electrophysiol 2017;3:1103-10. PUBMED | CROSSREF

99. Nguyen DT, Olson M, Zheng L, Barham W, Moss JD, Sauer WH. Effect of irrigant characteristics on lesion formation after radiofrequency energy delivery using ablation catheters with actively cooled tips. $J$ Cardiovasc Electrophysiol 2015;26:792-8. PUBMED | CROSSREF

100. Nguyen DT, Tzou WS, Sandhu A, et al. Prospective multicenter experience with cooled radiofrequency ablation using high impedance irrigant to target deep myocardial substrate refractory to standard ablation. JACC Clin Electrophysiol 2018;4:1176-85. PUBMED | CROSSREF

101. Chung FP, Vicera JJ, Lin YJ, et al. Clinical efficacy of open-irrigated electrode cooled with half-normal saline for initially failed radiofrequency ablation of idiopathic outflow tract ventricular arrhythmias. $J$ Cardiovasc Electrophysiol 2019;30:1508-16. PUBMED | CROSSREF

102. Sapp JL, Cooper JM, Soejima K, et al. Deep myocardial ablation lesions can be created with a retractable needle-tipped catheter. Pacing Clin Electrophysiol 2004;27:594-9. PUBMED | CROSSREF

103. Sapp JL, Beeckler C, Pike R, et al. Initial human feasibility of infusion needle catheter ablation for refractory ventricular tachycardia. Circulation 2013;128:2289-95. PUBMED | CROSSREF

104. Stevenson WG, Tedrow UB, Reddy V, et al. Infusion needle radiofrequency ablation for treatment of refractory ventricular arrhythmias. J Am Coll Cardiol 2019;73:1413-25. PUBMED | CROSSREF

105. Della Bella P, Peretto G, Paglino G, et al. Bipolar radiofrequency ablation for ventricular tachycardias originating from the interventricular septum: safety and efficacy in a pilot cohort study. Heart Rhythm. 2020 [Epub ahead of print]. PUBMED | CROSSREF

106. Igarashi M, Nogami A, Fukamizu S, et al. Acute and long-term results of bipolar radiofrequency catheter ablation of refractory ventricular arrhythmias of deep intramural origin. Heart Rhythm 2020;17:1500-7. PUBMED | CROSSREF

107. Futyma P, Santangeli P, Pürerfellner H, et al. Anatomic approach with bipolar ablation between the left pulmonic cusp and left ventricular outflow tract for left ventricular summit arrhythmias. Heart Rhythm 2020;17:1519-27. PUBMED | CROSSREF

108. Chang RJ, Stevenson WG, Saxon LA, Parker J. Increasing catheter ablation lesion size by simultaneous application of radiofrequency current to two adjacent sites. Am Heart J1993;125:1276-84. PUBMED | CROSSREF

109. Yang J, Liang J, Shirai Y, et al. Outcomes of simultaneous unipolar radiofrequency catheter ablation for intramural septal ventricular tachycardia in nonischemic cardiomyopathy. Heart Rhythm 2019;16:863-70. PUBMED | CROSSREF

110. Yamada T, Maddox WR, McElderry HT, Doppalapudi H, Plumb VJ, Kay GN. Radiofrequency catheter ablation of idiopathic ventricular arrhythmias originating from intramural foci in the left ventricular outflow tract: efficacy of sequential versus simultaneous unipolar catheter ablation. Circ Arrhythm Electrophysiol 2015;8:344-52. PUBMED | CROSSREF

111. van der Ree MH, Blanck O, Limpens J, et al. Cardiac radioablation - a systematic review. Heart Rhythm 2020;17:1381-92. PUBMED | CROSSREF 
112. Cuculich PS, Schill MR, Kashani R, et al. Noninvasive cardiac radiation for ablation of ventricular tachycardia. N Engl J Med 2017;377:2325-36.

PUBMED | CROSSREF

113. Neuwirth R, Cvek J, Knybel L, et al. Stereotactic radiosurgery for ablation of ventricular tachycardia. Europace 2019;21:1088-95.

PUBMED | CROSSREF

114. Loo BW Jr, Soltys SG, Wang L, et al. Stereotactic ablative radiotherapy for the treatment of refractory cardiac ventricular arrhythmia. Circ Arrhythm Electrophysiol 2015;8:748-50. PUBMED | CROSSREF

115. Robinson CG, Samson PP, Moore KM, et al. Phase I/II trial of electrophysiology-guided noninvasive cardiac radioablation for ventricular tachycardia. Circulation 2019;139:313-21. PUBMED | CROSSREF

116. Bhandari AK, Scheinman MM, Morady F, Svinarich J, Mason J, Winkle R. Efficacy of left cardiac sympathectomy in the treatment of patients with the long QT syndrome. Circulation 1984;70:1018-23. PUBMED | CROSSREF

117. Wilde AA, Bhuiyan ZA, Crotti L, et al. Left cardiac sympathetic denervation for catecholaminergic polymorphic ventricular tachycardia. N Engl J Med 2008;358:2024-9. PUBMED | CROSSREF

118. Murtaza G, Sharma SP, Akella K, et al. Role of cardiac sympathetic denervation in ventricular tachycardia: a meta-analysis. Pacing Clin Electrophysiol 2020;43:828-37. PUBMED | CROSSREF

119. Vaseghi M, Barwad P, Malavassi Corrales FJ, et al. Cardiac sympathetic denervation for refractory ventricular arrhythmias. J Am Coll Cardiol 2017;69:3070-80. PUBMED | CROSSREF

120. Vaseghi M, Gima J, Kanaan C, et al. Cardiac sympathetic denervation in patients with refractory ventricular arrhythmias or electrical storm: intermediate and long-term follow-up. Heart Rhythm 2014;11:360-6. PUBMED | CROSSREF

121. Meng L, Tseng CH, Shivkumar K, Ajijola O. Efficacy of stellate ganglion blockade in managing electrical storm: a systematic review. JACC Clin Electrophysiol 2017;3:942-9.

PUBMED | CROSSREF

122. Hawson J, Harmer JA, Cowan M, et al. Renal denervation for the management of refractory ventricular arrhythmias: a systematic review. JACC Clin Electrophysiol. 2020 [Epub ahead of print]. CROSSREF

123. Campbell T, Bennett RG, Garikapati K, et al. Prognostic significance of extensive versus limited induction protocol during catheter ablation of scar-related ventricular tachycardia. J Cardiovasc Electrophysiol 2020;31:2909-19.

PUBMED | CROSSREF

124. Soejima K, Stevenson WG, Maisel WH, Sapp JL, Epstein LM. Electrically unexcitable scar mapping based on pacing threshold for identification of the reentry circuit isthmus: feasibility for guiding ventricular tachycardia ablation. Circulation 2002;106:1678-83.

PUBMED | CROSSREF

125. Steven D, Reddy VY, Inada K, et al. Loss of pace capture on the ablation line: a new marker for complete radiofrequency lesions to achieve pulmonary vein isolation. Heart Rhythm 2010;7:323-30.

PUBMED | CROSSREF

126. Barbhaiya CR, Aizer A, Knotts R, et al. Simultaneous pace-ablate during CARTO-guided pulmonary vein isolation with a contact-force sensing radiofrequency ablation catheter. J Interv Card Electrophysiol 2019;54:119-24. PUBMED | CROSSREF 